\title{
A Power Counting Theorem for Feynman Integrals on the Lattice
}

\author{
Thomas Reisz ${ }^{\star}$ \\ Deutsches Elektronen-Synchrotron DESY, D-2000 Hamburg, Federal Republic of Germany
}

\begin{abstract}
A convergence theorem is proved, which states sufficient conditions for the existence of the continuum limit for a wide class of Feynman integrals on a space-time lattice. A new kind of a UV-divergence degree is introcduced, which allows the formulation of the theorem in terms of power counting conditions.
\end{abstract}

\section{Introduction}

Feynman integrals on a cubic, four-dimensional lattice have a very specific structure. In momentum space the integration domain is the Brillouin zone (BZ), hence compact for every non-vanishing lattice spacing $a$. Instead of being rational the integrand is a periodic function. If none of the propagators has vanishing mass, and so we will assume throughout this paper, a Feynman integral is absolutely convergent for every finite lattice spacing. We want to discuss the behaviour of such inegrals if the cutoff is removed, i.e., if the lattice spacing $a$ tends to zero.

There exists the well known power counting theorem of Hahn and Zimmermann [1] which states sufficient conditions for the absolute convergence of ordinary Feynman integrals. Convergence depends on the behavior of the integrand in various sections of the integration domain where some or all integration momenta get large. This behavior is described by use of UV-divergence degrees of the integrand with respect to so-called Zimmermann subspaces, i.e., special classes of affine subspaces of the integration momenta. If the divergence degrees with respect to all these subspaces are smaller than zero, the Feynman integral will be absolutely convergent. Unfortunately, this power counting theorem assumes a rational structure of the integrand and hence does not apply to diagrams with a lattice cutoff. Similar problems occur in connection with Weinberg's power counting theorem [2]. In fact, it is meaningless to discuss naively large momenta on the lattice, the integrand of a Feynman integral being periodic. Actually, if convergence holds,

* Address after September 1987: Max Planck Institut für Physik und Astrophysik, D-8000 München 40, Federal Republic of Germany 
only a neighborhood of zero momentum in the Brillouin zone should contribute to the continuum limit. Other contributions should vanish.

At first sight it seems reasonable to assume existence of the continuum limit of a lattice Feynman integral if the naive $a \rightarrow 0$-limit of the integrand is integrable. A simple counter-example shows that this is not so. Consider the one-dimensional integral

$\hat{I}(q ; \mu, a)=\int_{-\pi / a}^{\pi / a} d t \frac{\left(\frac{2}{a} \sin \frac{t a}{2}\right)^{4}\left[\cos ^{2} \frac{q a}{2}-\cos ^{2} \frac{t a}{2}\right]+\left(\frac{2}{a} \sin \frac{t a}{2}\right)^{2} \cos ^{2} \frac{q a}{2}\left(\frac{2}{a} \sin \frac{q a}{2}\right)^{2}}{\left(\frac{4}{a^{2}} \sin ^{2} \frac{t a}{2}+\mu^{2}\right)^{2}}$

The formal continuum limit is given by

$$
\int_{-\infty}^{\infty} d t \frac{t^{2} q^{2}}{\left(t^{2}+\mu^{2}\right)^{2}},
$$

which is absolutely convergent, whereas expanding $\cos ^{2}(q a / 2)$ yields

$$
\hat{I}(q ; \mu, a)=\frac{16}{a} \int_{-\pi}^{\pi} d t \frac{\sin ^{4} \frac{t}{2}\left[1-\cos ^{2} \frac{t}{2}\right]}{\left(4 \sin ^{2} \frac{t}{2}+\mu^{2} a^{2}\right)^{2}}+O(1)
$$

for small $a$, i.e., $\hat{I}$ is (linearly) divergent. This example shows that "continuum UVdegrees" do not suffice to control continuum limit behavior of Feynman integrals on the lattice. This means we have to take into account the lattice structure more carefully. This can be done by introducing a new kind of UV-degrees which we shall call "lattice UV-degrees." It will be shown that they are suited to describe correctly the leading term in a small $a$ expansion of Feynman integrals. In some sense, these degrees describe the behavior of Feynman integrals at large momenta and small lattice spacing simultaneously. Using them, we formulate a power counting theorem on the lattice which states existence of the continuum limit if all lattice UVdegrees are smaller than zero. In a forthcoming paper this theorem will be used to construct a general renormalization procedure for lattice field theories.

This article is essentially divided into two parts. The first part is devoted to the lattice power counting theorem. In Sect. 2 we introduce the notion of a lattice UVdegree for functions containing a wide class of Feynman integrands on the lattice. We show that almost all properties one does expect of a degree are satisfied. In Sect. 3 the power counting theorem is formulated, and the first steps of the proof are done in Sect. 4. As will be seen the numerator of the integrand causes some technical problems, but the integral is always bounded by a sum of generalized continuum Feynman integrals. These are integrals which have a structure similar to Feynman integrals in the continuum, but with a sharp cutoff and a more complicated numerator. Hence it is necessary to have a theorem which states the cutoff dependence of such integrals. Such an auxiliary power counting theorem is formulated in Sect. 5, and in Sect. 6 it is shown that the numerator of a lattice 
Feynman integrand admits an estimate which allows application of this auxiliary theorem to complete the proof of the power counting theorem in Sect. 7. The second part of this paper is devoted to the proof of the auxiliary power counting theorem. Section 8 contains technical lemmas, and in Sect. 9 the proof is given by induction on the number of loops.

\section{UV-Degrees on the Lattice}

We shall consider momentum space-integrals of the general form

where

$$
\hat{I}(q ; \mu, a)=\int_{-\pi / a}^{\pi / a} d^{4} k_{1} \ldots d^{4} k_{m} \frac{V(k, q ; \mu, a)}{C(k, q ; \mu, a)}
$$

$$
\begin{gathered}
C(k, q ; \mu, a)=\prod_{i=1}^{n}\left(\frac{\eta_{i}\left(l_{i} a\right)}{a^{2}}+\mu_{i}^{2}\right), \quad \mu_{i}^{2}>0, \\
l_{i}(k, q)=K_{i}(k)+Q_{i}(q)=\sum_{j=1}^{m} C_{i j} k_{j}+Q_{i}(q), \quad i=1, \ldots, n,
\end{gathered}
$$

and the $Q_{i}$ are linear ( $q$ represents the external momenta and $k$ the loop momenta). $V$ and $\eta$ are functions to be specified below. As explained in the introduction, to discuss the behavior of the integral when the cutoff is removed, it does not suffice to consider the continuum limit of the integrand only. We will now define special classes of functions and for them a generalized notion of a UV-degree. These degrees allow a generalization of the old power counting theorem [1] which can be applied to diagrams with a lattice cutoff.

We shall consider functions of the lattice spacing $a>0$, of "external" momenta $w$ and "internal" momenta $u$.

Definition 2.1. For arbitrary $m \in \mathbf{Z}$, we define $\mathscr{C}_{m}$ to be the set of functions $V$ in real variables $(u, w)=\left(u_{1}, \ldots, u_{h}\right),\left(w_{1}, \ldots, w_{s}\right)$ and $a>0$ of the form

$$
V(u, w ; a)=\left(1 / a^{m}\right) F(u a, w a),
$$

$$
F \in C^{\infty} \text {. }
$$

$\mathscr{C}$ is defined as the set of functions which are finite sums of functions in some $\mathscr{C}_{m}$.

$C^{\infty}$ is the set of infinitely often differentiable functions. To simplify the notation, we shall use multi-indices. Set $\mathbf{N}_{0}=\mathbf{N} \cup\{0\}=\{0,1,2, \ldots\}$. For $b \in \mathbf{N}_{0}^{n}$ and $u \in \mathbf{R}^{n}$ define

$$
b !=b_{1} ! \ldots b_{n} !, \quad u^{b}=u_{1}^{b_{1}} \ldots u_{n}^{b_{n}}, \quad|b|=\sum_{i=1}^{n} b_{i} .
$$

The well known definition of a UV-degree of polynomials is given in Appendix C. We now define the lattice version of a UV-degree of a function $V \in \mathscr{C}_{m}$ with respect to internal momenta $u$. 
Definition 2.2. Let $V \in \mathscr{C}_{m}$ be of the form (2.2) and $r_{u}$ the largest non-negative integer such that

$$
\left(\frac{\partial^{b}}{\partial w^{b}} F(u, w)\right)_{w=0} \equiv 0 \quad \text { for all } \quad b \in \mathbf{N}_{0}^{s}, \quad|b|<r_{u} .
$$

Then the UV-degree of $V$ with respect to $(u)$ is defined by

$$
\overline{\operatorname{degr}}_{\hat{u}} V=m-r_{u} .
$$

The UV-degree of a function $V \in \mathscr{C}_{m}$ with respect to internal momenta $u$ is determined by the asymptotic behavior of $V$ for small external momenta $w$. Note that always $\overline{\operatorname{degr}}_{\hat{u}} V \leqq m$. With respect to all variables, $\overline{\operatorname{degr}}_{\hat{u} w} V=m$, the set of complementary variables being empty. If for all $b$

$$
\left.\frac{\partial^{b} F(u, w)}{\partial w^{b}}\right|_{w=0} \equiv 0
$$

we set $\overline{\operatorname{degr}}_{\hat{u}} V=-\infty$. Note that, contrary to the definition of a polynomial degree, we never fix external momenta.

This form of a degree will be useful later in many circumstances, e.g. in proving convergence of renormalized Feynman integrals. An equivalent, even simpler definition is the following. Let $V \in \mathscr{C}_{m}$ for some $m \in \mathbf{Z}$. Then $\delta_{a}=\overline{\operatorname{degr}}_{\hat{u}} V$ if and only if

$$
V\left(\lambda u, w ; \frac{1}{\lambda} a\right)=A(u, w ; a) \lambda^{\delta_{u}}+O\left(\lambda^{\delta_{u}-1}\right), \quad \lambda \rightarrow \infty
$$

where $A(u, w ; a) \neq 0$ ( $A$ is a polynomial in $w$ and $C^{\infty}$ in $u$ ).

As can be seen from (2.4), the UV-degree of $V$ with respect to $u$ is determined by the behavior of $V$ for large $u$ and small $a$ simultaneously. There may be high powers in $\lambda$ not occurring in the large $u$ behavior of the leading term of a small $a$ expansion of $V$. For example, let

$$
V(t, q ; a)=\left(\frac{2}{a} \sin \frac{t a}{2}\right)^{4}\left[\cos ^{2} \frac{q a}{2}-\cos ^{2} \frac{t a}{2}\right]+\left(\frac{2}{a} \sin \frac{t a}{2}\right)^{2} \cos ^{2} \frac{q a}{2}\left(\frac{2}{a} \sin \frac{q a}{2}\right)^{2}
$$

as in the introduction. Then $\overline{\operatorname{degr}}_{\hat{t}} V=4$, but $\lim _{a \rightarrow 0} V(\lambda t, q ; a)=\lambda^{2} t^{2} q^{2}$. The leading term does not show the correct asymptotic behaviour of $V$ if $t$ and $1 / a$ tend to infinity simultaneously.

If $F$ in Definition 2.2 is a homogeneous polynomial in $u, w$ of degree $m_{0}$, then

$$
V(u, w ; a)=a^{d} F(u, w) \text { and } \overline{\operatorname{degr}}_{u} F=m_{0}-r_{u}=d+\overline{\operatorname{degr}}_{\hat{u}} V,
$$

where $d=m_{0}-m$. In this case the lattice degree reproduces the old polynomial degree up to a constant which counts inverse powers of the lattice spacing. Every additional factor $a$ in $V$ decreases the lattice degree by one, i.e., improves the continuum limit behavior.

We now generalize Definition 2.2 to functions in $\mathscr{C}$. 

Definition 2.3. Let $V \in \mathscr{C}, V=\sum_{i \in I} V_{i}, V_{i} \in \mathscr{C}_{m_{\imath}}$ for some $m_{i} \in \mathbf{Z}, m_{i} \neq m_{k}$ for $i \neq k$. Then
we define

$$
\overline{\operatorname{degr}}_{\hat{u}} V=\max _{i \in I} \overline{\operatorname{degr}}_{\hat{u}} V_{i}
$$

By Definition 2.3, the UV-degree is uniquely defined for every $V \in \mathscr{C}$. Again, $\delta_{u}=\overline{\operatorname{degr}}_{\hat{u}} V$ if and only if $V$ shows a behavior (2.4). The lattice degree defined in this way has quite similar properties as the usual degree of a polynomial. Using (2.4), we get

Lemma 2.1. Let $V, V_{1}, \ldots, V_{p} \in \mathscr{C}$ be functions in variables $(u, w)$ and $a>0$. Then

$$
\begin{aligned}
& \overline{\operatorname{degr}}_{\hat{u}} \sum_{i=1}^{p} V_{i} \leqq \max _{i=1, \ldots, p} \overline{\operatorname{degr}}_{\hat{u}} V_{i}, \\
& \overline{\operatorname{degr}}_{\hat{u}} \prod_{i=1}^{p} V_{i} \leqq \sum_{i=1}^{p} \overline{\operatorname{degr}_{\hat{u}}} V_{i}, \\
& \overline{\operatorname{degr}}_{\hat{u}} \frac{\partial^{c}}{\partial u^{c}} V \leqq \overline{\operatorname{degr}}_{\hat{u}} V-|c|, \\
& \overline{\operatorname{degr}}_{\hat{u}} \frac{\partial^{c}}{\partial w^{c}} V \leqq \overline{\operatorname{degr}}_{\hat{u}} V .
\end{aligned}
$$

Note that the second statement is an inequality, whereas the analogous property of polynomial degrees is an equality.

We further restrict the function classes $\mathscr{C}_{m}$ and $\mathscr{C}$. Until now we have not made any assumption about the behavior of functions in $\mathscr{C}$ for small lattice spacing $a$. We now assume existence of the continuum limit.

Definition 2.4. $\mathscr{C}_{m}^{c}$ is the set of functions $V$ defined by

a) $V \in \mathscr{C}_{m}$,

b) $\lim _{a \rightarrow 0} V(u, w ; a)$ exists.

$\mathscr{C}^{c}$ consists of all finite sums of functions in some $\mathscr{C}_{m}^{c}$.

This roughly defines the class of functions to which numerators and denominators of Feynman integrals belong. In particular, they are assumed to be infinitely often differentiable, and their continuum limit exists. Before defining the exact form of Feynman integrals to which the power counting theorem will be applicable we state some important properties of the class $\mathscr{C}_{m}^{c}$. If $V \in \mathscr{C}_{m}^{c}$ is independent of momenta $u$, then

$$
\overline{\operatorname{degr}}_{\hat{u}} V(u, w ; a) \leqq 0 .
$$

If in addition $\lim _{a \rightarrow 0} V(u, w ; a) \neq 0$, we have

$$
\overline{\operatorname{degr}}_{\hat{u}} V(u, w ; a)=0 .
$$


Every $V \in \mathscr{C}_{m}^{c}$ has an expansion for small lattice spacing $a$ of the form

$$
V(u, w ; a)=\frac{1}{a^{m}} F(u a, w a)=P(u, w)+R(u, w ; a) .
$$

The continuum limit $P$ of $V$ is a homogeneous polynomial of order $m$. In general,

$$
\overline{\operatorname{degr}}_{u} P \leqq \overline{\operatorname{degr}}_{\hat{u}} V .
$$

As shown by the example considered above, $\overline{\operatorname{degr}}_{u} P<\overline{\operatorname{degr}}_{\hat{u}} V$ cannot be excluded. However, with respect to all momenta $u, w$,

$$
\overline{\operatorname{degr}}_{u w} P=\overline{\operatorname{degr}}_{\widehat{u w}} V \quad \text { if } P(u, w) \neq 0 .
$$

In this special case the lattice degree is determined by the continuum limit. In general, "lattice effects" are described by the remainder $R(u, w ; a)$. As can easily be seen, $R$ admits an estimate

$$
|R(u, w ; a)| \leqq a^{p} \sum_{i \in I}\left|Q_{i}(u, w)\right|, \quad p \geqq 1,
$$

where $I$ is a finite set and $Q_{i}$ are polynomials satisfying $\overline{\operatorname{degr}}_{u} Q_{i} \leqq \overline{\operatorname{degr}}_{\hat{u}} V+p$. This means every additional power of $u$ in $Q_{i}$ (with respect to $\overline{\operatorname{degr}_{\hat{u}} V}$ ) is accompanied by a power of $a$. Unfortunately, (2.12) depends on the partition of $(u, w)$ into internal and external momenta. Later we will derive a much more general inequality which allows determination of the cutoff dependence of Feynman integrals having such a $V$ as the numerator of the integrand.

We now define a class of Feynman integrands on the lattice. To this end we choose momentum variables $\left(u_{1}, \ldots, u_{h}\right)$ and $\left(w_{1}, \ldots, w_{s}\right)$, where $u_{i}$ and $w_{j}$ are fourmomenta. The following considerations can easily be extended to other dimensions.

Definition 2.5. $\mathscr{F}$ is the set of functions $F$ in momentum variables $\left(u_{1}, \ldots, u_{h}\right)$, $\left(w_{1}, \ldots, w_{s}\right)$, masses $\mu=\left(\mu_{1}, \mu_{2}, \ldots\right)$ and $a>0$ of the form

$$
F=\frac{V}{C}
$$

and the following properties:

1. $V \in \mathscr{C}^{c}$ is of the form $V(u, w ; \mu, a)=\sum_{i \in I} P_{i}(\mu) V_{i}(u, w ; a), I$ a finite set and $V_{i} \in \mathscr{C}_{m_{i}}^{c}, m_{i} \in \mathbf{Z}$. For every $i \in I, P_{i}$ is a polynomial in the masses $\mu{ }^{1}$

2. $C$ is a product

$$
C=\prod_{i=1}^{n}\left[e_{i}\left(l_{i} ; a\right)+\mu_{i}^{2}\right],
$$

where $n \in \mathbf{N}_{0}=\{0,1,2, \ldots\}$. The "line momenta" $l_{i} \neq 0$ are of the form

$$
l_{i}(u, w)=\sum_{j=1}^{h} c_{i j} u_{j}+\sum_{k=1}^{s} d_{i k} w_{k},
$$

\footnotetext{
${ }^{1}$ The mass dependence of the numerator is important if Feynman integrals containing massless propagators are to be renormalized. Then it will be necessary to introduce auxiliary masses
} 
where $c_{i j}, d_{i k}$ are real constants, and

where

$$
e_{i} \in \mathscr{C}_{2}^{\mathrm{c}}, \quad e_{i}\left(l_{i} ; a\right)=\frac{1}{a^{2}} \eta_{i}\left(l_{i} a\right)
$$

$$
\eta_{i}\left(l_{i} a \neq 0\right)>0 \text { for } l_{i} \in[-\pi / a, \pi / a]^{4},
$$

and

$$
\eta_{i} 2 \pi \text {-periodic in every component of } l_{i} a \text {, }
$$

$$
\lim _{a \rightarrow 0} e_{i}\left(l_{i} ; a\right)=l_{i}^{2}
$$

With respect to addition and multiplication, the set of functions $\mathscr{F}$ is closed. Furthermore, $\mathscr{F}$ is invariant under differentiation. We always assume that every $e_{i}\left(l_{i} ; a\right)$ is periodic in $l_{i}$ with the $\mathrm{BZ}$, and $e_{i}$ should have only one zero in the BZ, located at vanishing momentum. Especially, naive fermions are excluded, their propagators having more than one pole. If we would drop this condition, our general assumptions about the form of the numerator would not be sufficient to get convergence of a Feynman integral in the continuum limit.

For $F=V / C \in \mathscr{F}$ we define

Note that

$$
\overline{\operatorname{degr}}_{\hat{u}} F=\overline{\operatorname{degr}}_{\hat{u}} V-\overline{\operatorname{degr}}_{\hat{u}} C
$$

$$
\overline{\operatorname{degr}}_{\hat{u}}\left(e_{i}\left(l_{i} ; a\right)+\mu_{i}^{2}\right)= \begin{cases}2 & \text { if }\left(c_{i 1}, \ldots, c_{i n}\right) \neq 0 \\ 0 & \text { otherwise },\end{cases}
$$

hence for $F=V / C \in \mathscr{F}$

$$
\overline{\operatorname{degr}}_{\hat{u}} F=\overline{\operatorname{degr}}_{\hat{u}} V-2 n_{u},
$$

where $n_{u}$ is the number of line momenta $l_{i}, i \in\{1, \ldots, n\}$, which are dependent on $u$. In particular

$$
\overline{\operatorname{degr}_{\hat{u}}} \prod_{i=1}^{n}\left[e_{i}\left(l_{i} ; a\right)+\mu_{i}^{2}\right]=\sum_{i=1}^{n} \overline{\operatorname{degr}_{\hat{u}}}\left[e_{i}\left(l_{i} ; a\right)+\mu_{i}^{2}\right] .
$$

In this special case Lemma 2.1.2 is an equality, i.e., the UV-degree of the denominator is already given by the polynomial degree of its continuum limit. As a consequence, for every $F \in \mathscr{F}$

$$
\overline{\operatorname{degr}}_{u} \lim _{a \rightarrow 0} F(u, w ; \mu, a) \leqq \overline{\operatorname{degr}}_{\hat{u}} F(u, w ; \mu, a)
$$

The UV-degrees of functions $F \in \mathscr{F}$ have "typical" degree properties. They are direct consequences of Definition 2.5 and of Lemma 2.1, and are listed below, although we do not make use of them in this paper. Nevertheless, they are of importance, especially in proving convergence of renormalization schemes [4].

Lemma 2.2. Let $F, F_{1}, \ldots, F_{p} \in \mathscr{F}$. Then

$$
\overline{\operatorname{degr}}_{\hat{u}} \sum_{i=1}^{p} F_{i} \leqq \max _{i=1, \ldots, p} \overline{\operatorname{degr}}_{\hat{u}} F_{i}
$$


2.

$$
\overline{\operatorname{degr}}_{\hat{u}} \prod_{i=1}^{p} F_{i} \leqq \sum_{i=1}^{p} \overline{\operatorname{degr}}_{\hat{u}} F_{i}
$$

3.

$$
\overline{\operatorname{degr}}_{\hat{u}} \frac{\partial^{c}}{\partial u^{c}} F \leqq \overline{\operatorname{degr}}_{\hat{u}} F-|c|
$$

4.

$$
\overline{\operatorname{degr}}_{\hat{u}} \frac{\partial^{c}}{\partial w^{c}} F \leqq \overline{\operatorname{degr}}_{\hat{u}} F
$$

For instance, to prove 3 , let $F \in \mathscr{F}$. Then

hence

$$
\frac{\partial}{\partial u} F=\frac{C \frac{\partial V}{\partial u}-V \frac{\partial C}{\partial u}}{C^{2}}
$$

$$
\begin{aligned}
\overline{\operatorname{degr}}_{\hat{u}} \frac{\partial F}{\partial u} & \leqq \max \left(\overline{\operatorname{degr}}_{\hat{u}} C \frac{\partial V}{\partial u}, \overline{\operatorname{degr}}_{\hat{u}} V \frac{\partial C}{\partial u}\right)-2 \overline{\operatorname{degr}}_{\hat{u}} C \\
& \leqq \overline{\operatorname{degr}}_{\hat{u}} V-\overline{\operatorname{degr}}_{\hat{u}} C-1=\overline{\operatorname{degr}}_{\hat{u}} F-1 .
\end{aligned}
$$

The assertion now follows by induction on the number of derivatives.

\section{The Power Counting Theorem}

We consider Feynman integrals

where

$$
\hat{I}(q, \mu, a)=\int_{-\pi / a}^{\pi / a} d^{4} k_{1} \ldots d^{4} k_{m} F(k, q ; \mu, a)
$$

$$
F(k, q ; \mu, a)=V(k, q ; \mu, a) / C(k, q ; \mu, a) \in \mathscr{F} .
$$

We assume periodicity of the numerator $V \in \mathscr{C}^{c}$ with the Brillouin zone $[-\pi / a, \pi / a]^{4}$ in all internal momenta $k_{1}, \ldots, k_{m}$. An important notion is given by the following Definition 3.1. Let $\mathscr{L}$ be a set of four-vectors $l_{i}$,

$$
l_{i}(k, q)=K_{i}(k)+Q_{i}(q)=\sum_{j=1}^{m} C_{i j} k_{j}+Q_{i}(q), \quad i=1, \ldots, N, \quad C_{i j} \in \mathbf{R} .
$$

$\mathscr{L}$ is called natural with respect to $k$, if the following conditions hold:

1.

$$
\begin{aligned}
C_{i j} \in \mathbf{Z} \quad \text { for all } i=1, \ldots, N ; j=1, \ldots, m, \\
\\
\operatorname{rank}\left(C_{i j}\right)=m, \\
\left(C_{i 1}, \ldots, C_{i m}\right) \neq 0 \quad \text { for all } i=1, \ldots, N .
\end{aligned}
$$

2. If the four-momenta

$$
k_{l}^{\prime}=\sum_{j=1}^{m} C_{i_{1} j} k_{j}, \quad l=1, \ldots, m
$$


are linearly independent, then

$$
k_{j}=\sum_{l=1}^{m} A_{j l} k_{l}^{\prime} \text { with } A_{j l} \in \mathbf{Z} .
$$

This condition is natural in the sense that arbitrary independent $l_{i} \in \mathscr{L}$ could be chosen as integration momenta, the coefficients $C_{i j}$ always being integer-valued. For a Feynman integral this condition is ensured if all loop momenta $k_{1}, \ldots, k_{m}$ coincide with momenta of lines up to external momenta [3]. Also, using periodicity of the integrand, the integration domain could always be chosen to be $[-\pi / a, \pi / a]^{4 m}$. As example of the importance of line momenta to be natural consider

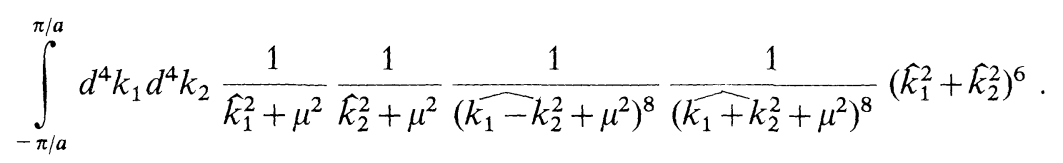

Here $\hat{k}^{2}=\sum_{\mu=1}^{4}\left(4 / a^{2}\right) \sin ^{2}\left(k_{\mu} a / 2\right)$ and $\mu^{2}>0$. All criteria of the power counting theorem below are satisfied except that the set $\left\{k_{1}, k_{2}, k_{1}-k_{2}, k_{1}+k_{2}\right\}$ is not natural. In fact, if $0<\varepsilon<1 / 2$ and $e=(1,0,0,0)$, the integral is divergent in the sector

$$
\left\|k_{1}\right\| \geqq \frac{\pi}{a} \varepsilon,\left\|k_{2}\right\| \geqq \frac{\pi}{a} \varepsilon,\left\|k_{1}+k_{2}-\frac{2 \pi}{a} e\right\|<\frac{\pi}{a} \varepsilon,\left\|k_{1}-k_{2}\right\|<\frac{\pi}{a} \varepsilon
$$

as $a \rightarrow 0$ where $\|l\|=\sqrt{\sum_{i=1}^{4} l_{i}^{2}}$ for $l=\left(l_{1}, \ldots, l_{4}\right) \in \mathbf{R}^{4}$. As will be seen below, naturalness means that line momenta in neighborhoods of poles ${ }^{2}$ of propagators in higher BZs can be transformed simultaneously into neighborhoods of the poles in the first BZ by translation with reciprocal lattice vectors. Under such a transformation the numerator of a Feynman integrand is invariant. This would not be the case by other translations. They would produce explicit negative powers in the lattice spacing destroying convergence.

Before defining UV-divergence degrees of Feynman integrals in Zimmermann subspaces, we have to introduce the notion of a basis of a set of line momenta. Given variables $k=\left(k_{1}, \ldots, k_{m}\right)$ (loop momenta) and $q=\left(q_{1}, \ldots, q_{M}\right)$ (external momenta), $k_{i}, q_{j} \in \mathbf{R}^{4}$, let $L$ denote the space of all linear mappings $l: \mathbf{R}^{4 m} \times \mathbf{R}^{4 M} \rightarrow \mathbf{R}^{4}$ of the form

$$
\begin{aligned}
l(k, q) & =K(k)+Q(q), \\
K(k) & =\sum_{i=1}^{m} a_{i} k_{i} ; \quad a_{i} \in \mathbf{R}, \quad i=1, \ldots, m, \\
Q(q) & =\sum_{j=1}^{M} b_{j} q_{j} ; \quad b_{j} \in \mathbf{R}, \quad j=1, \ldots, M
\end{aligned}
$$

in the four-momenta $k_{1}, \ldots, k_{m}$ and $q_{1}, \ldots, q_{M} . K$ and $Q$ is said to be linear in $k$ and $q$, respectively.

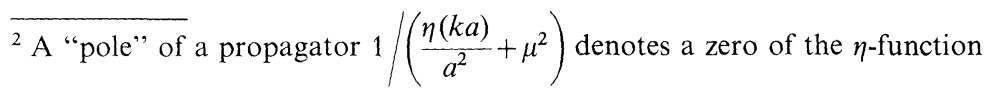


Let $\mathscr{M} \subseteq L$ be an arbitrary subset. Elements $l_{1}, \ldots, l_{s} \in \mathscr{M}$,

$$
l_{i}(k, q)=\sum_{j=1}^{m} a_{i j} k_{j}+\sum_{j=1}^{M} b_{i j} q_{j}, \quad i=1, \ldots, s,
$$

are called linearly independent with respect to $k$ if their homogeneous parts in $k$ are linearly independent. Furthermore, $\left\{l_{1}, \ldots, l_{s}\right\} \subseteq \mathscr{M}$ is called a basis of $\mathscr{M}$ with respect to $k$ if $l_{1}, \ldots, l_{s}$ are linearly independent and every $l \in \mathscr{M}$ can be written as

$$
l(k, q)=\sum_{i=1}^{s} c_{i} l_{i}(k, q)+Q(q)
$$

where $c_{i} \in \mathbf{R}, i=1, \ldots, s$ and $Q$ is linear. In this case we define $\operatorname{rank}_{k} \cdot \mathscr{M} \equiv s$.

We now define UV-divergence degrees with respect to Zimmermann subspaces. Let $\mathscr{L}$ be a natural set of four-momenta ${ }^{3}$ and

$$
\begin{gathered}
u_{1}=l_{i_{1}}, \ldots, u_{d}=l_{i_{d}}, \\
v_{1}=l_{j_{1}}, \ldots, v_{m-d}=l_{j_{m-d}}
\end{gathered}
$$

be an arbitrary basis of $\mathscr{L}$ with respect to $k, 1 \leqq d \leqq m$. By fixing $v_{1}, \ldots, v_{m-d}$ we define a class $H$ of affine subspaces in the space of integration momenta $\mathbf{R}^{4 m} . H$ is called a Zimmermann subspace, $(u)=\left(u_{1}, \ldots, u_{d}\right)$ is called the parametrization of $H$, and $(v)=\left(v_{1}, \ldots, v_{m-d}\right)$ are the complementary parameters of $H$. For $F \in \mathscr{F}$ we define

$$
\overline{\operatorname{degr}}_{\hat{H}} \hat{I}(q ; \mu, a)=4 d+\overline{\operatorname{degr}}_{\hat{u}} F(k(u, v, q), q ; \mu, a)
$$

$(v, q$ represent the "external momenta" of $H)$. The set of all Zimmermann subspaces, for all bases (3.7), will be denoted by $\mathscr{H}$. Note that $\mathscr{H}$ depends on the set $\mathscr{L}$ of four-momenta. Now we state

\section{Theorem 1. Power Counting Theorem. Let}

$$
\hat{I}(q ; \mu, a)=\int_{-\pi / a}^{\pi / a} d^{4} k_{1} \ldots d^{4} k_{m} F(k, q ; \mu, a)
$$

and $F \in \mathscr{F}$ of the form

$$
F(k, q ; \mu, a)=\frac{V(k, q ; \mu, a)}{C(k, q ; \mu, a)},
$$

where $V \in \mathscr{C}^{c}$ is $(2 \pi / a)$-periodic in every component of $k_{1}, \ldots, k_{m}$, and

$$
C(k, q ; \mu, a)=\prod_{i=1}^{n}\left[e_{i}\left(l_{i}(k, q) ; a\right)+\mu_{i}^{2}\right], \quad \mu_{i}^{2}>0 .
$$

Let $\mathscr{L}$ be a natural set of four-momenta and $\left\{l_{1}, \ldots, l_{n}\right\} \subseteq \mathscr{L}$. If for every $H \in \mathscr{H}$,

$$
\overline{\operatorname{degr}}_{\hat{H}} \hat{I}(q ; \mu, a)<0,
$$

\footnotetext{
${ }^{3}$ Actually, property 1 in the definition of naturalness would be sufficient to define UV-degree of Feynman integrals. However it is convenient here to assume $\mathscr{L}$ to be natural, this being an important assumption of the power counting theorem
} 
the continuum limit of $\hat{I}(q ; \mu, a)$ exists and is given by

where

$$
\lim _{a \rightarrow 0} \hat{I}(q ; \mu, a)=\int_{-\infty}^{\infty} d^{4} k_{1} \ldots d^{4} k_{m} \frac{P(k, q, \mu)}{E(k, q, \mu)}
$$

$$
\begin{aligned}
& P(k, q, \mu)=\lim _{a \rightarrow 0} V(k, q ; \mu, a), \\
& E(k, q, \mu)=\lim _{a \rightarrow 0} C(k, q ; \mu, a) .
\end{aligned}
$$

If $P \neq 0$, the set $\mathscr{L}^{\prime}=\left\{l_{1}, \ldots, l_{n}\right\}$ contains a basis of $\mathscr{L}$ with respect to $k$ [otherwise $\omega(H) \geqq 0$ for some $H \in \mathscr{H}]$. Hence, if $\mathscr{L}$ is natural so is $\mathscr{L}^{\prime}$. In this case the theorem can be formulated using $\mathscr{L}^{\prime}$ instead of $\mathscr{L}$. The continuum limit (3.10) is absolutely convergent according to (2.16) and the power counting theorem of Y. Hahn and W. Zimmermann [1] (or by Theorem 2 below).

As an example for the importance of (3.9), let us look at (1.1) in the introduction again. Only one subspace must be considered, and the corresponding divergence degree is equal to one. Hence the theorem cannot be applied, and as we have seen, (1.1) is in fact divergent in the limit of vanishing lattice spacing $a$.

To prove the theorem, using Definition 2.3, it does suffice to assume $V \in \mathscr{C}_{m_{0}}^{c}$ for some $m_{0} \in \mathbf{Z}$. Hence let us consider

$$
\hat{I}(q ; \mu, a)=\int_{-\pi / a}^{\pi / a} d^{4} k_{1} \ldots d^{4} k_{m} \frac{V(k, q ; a)}{\prod_{i=1}^{n}\left[\frac{\eta_{i}\left(l_{i} a\right)}{a^{2}}+\mu_{i}^{2}\right]},
$$

$V \in \mathscr{C}_{m_{0}}^{c}, m_{0} \in \mathbf{Z}$. Without loss of generality we assume $\mathscr{L}=\left\{l_{1}, \ldots, l_{N}\right\}, N$ an integer greater or equal to $n$, and that $k_{1}, \ldots, k_{m}$ are contained in $\mathscr{L}$. By naturalness of $\mathscr{L}$, this is always possible to arrange by a linear transformation.

\section{Proof of the Power Counting Theorem : First Steps}

The proof idea is as follows: The integral (3.11) will be written as a sum of integrals over various sections in momentum space. The division of the integration domain will be done in dependence on the configuration of line momenta $l_{i}$. For every propagator we distinguish line momentum in neighborhoods of the poles and outside of them. As will be seen, a propagator can then be estimated by its continuum limit or by some powers of the lattice spacing $a$, respectively.

For $l \in \mathbf{R}^{4}$ define

$$
\Theta_{\varepsilon}(l)= \begin{cases}0 & \text { if }\|l-(2 \pi / a) z\|<(\pi / a) \varepsilon \text { for some } z \in \mathbf{Z}^{4} \\ 1 & \text { otherwise },\end{cases}
$$

where $\varepsilon$ is a small positive constant which will be chosen below. Using Heavisides $\Theta$-function, $\Theta(x)=1$ if $x \leqq 0$ and $\Theta(x)=0$ if $x<0$,

$$
1=\Theta_{\varepsilon}(l)+\sum_{z \in \mathbf{Z}^{4}} \Theta\left(\frac{\pi}{a} \varepsilon-\left\|l-\frac{2 \pi}{a} z\right\|\right) .
$$


Doing so for every propagator, (3.11) can be written as

$$
\hat{I}(q ; \mu, a)=\sum_{J \subseteq\{1, \ldots, n\}} \sum_{z} \hat{I}_{J z}(q ; \mu, a)
$$

where for every "sector" $J, z=\left(z_{i} \mid i \in J\right)$, we have

$$
\begin{aligned}
\hat{I}_{J z}(q ; \mu, a)= & \int_{-\pi / a}^{\pi / a} d^{4} k_{1} \ldots d^{4} k_{m} \frac{V(k, q ; a)}{\prod_{i=1}^{n}\left(\frac{\eta_{i}\left(l_{i} a\right)}{a^{2}}+\mu_{i}^{2}\right)} \\
& \cdot\left(\prod_{i \in J} \Theta\left(\frac{\pi}{a} \varepsilon-\left\|l_{i}-\frac{2 \pi}{a} z_{i}\right\|\right) \cdot \prod \Theta_{\varepsilon}\left(l_{i}\right),\right.
\end{aligned}
$$

and for every $J$ the sum $\sum_{z}$ runs over finitely many configurations $z$. We have to estimate the contributions of all integrals (4.4) for small lattice spacing $a$. To this end, we make an appropriate transformation for each integral (4.4). As a consequence of naturalness of the set $\mathscr{L}$ of four-momenta, for small enough $\varepsilon>0$ and for every $J, z$, there exists a translation

$$
k_{j} \rightarrow k_{j}+\frac{2 \pi}{a} \delta_{j}, \quad \delta_{j} \in \mathbf{Z}^{4}, \quad j=1, \ldots, m,
$$

so that

$$
l_{i} \rightarrow l_{i}+\frac{2 \pi}{a} z_{i} \text { for all } i \in J
$$

This is shown in Appendix D. By (4.5), all line momenta at poles of propagators in higher BZs are shifted into neighborhoods of the origin in the first BZ, leaving $V(k, q ; a)$ and all $\eta_{i}$ invariant. Consequently

$$
\begin{aligned}
\hat{I}_{J z}(q ; \mu, a)= & \int_{\sigma_{J}} d^{4} k_{1} \ldots d^{4} k_{m} \frac{V(k, q ; a)}{\prod_{i=1}^{n}\left(\frac{\eta_{i}\left(l_{i} a\right)}{a^{2}}+\mu_{i}^{2}\right)} \\
& \cdot\left(\prod_{i \in J} \Theta\left(\frac{\pi}{a} \varepsilon-\left\|l_{i}\right\|\right)\right) \cdot \prod_{i \neq J} \Theta_{\varepsilon}\left(l_{i}\right),
\end{aligned}
$$

where

$$
\begin{gathered}
\sigma_{J}=\left\{\left(k_{1}, \ldots, k_{m}\right) \in \mathbf{R}^{4 m} \mid-\left(1+2\left(\delta_{j}\right)_{i}\right) \pi / a \leqq\left(k_{j}\right)_{i} \leqq\left(1-2\left(\delta_{j}\right)_{i}\right) \pi / a,\right. \\
j=1, \ldots, m ; \quad i=1, \ldots, 4\} .
\end{gathered}
$$

Now, in every integral (4.6) the propagators can easily be estimated, using their properties listed in Definition 2.5. Again, for small enough $\varepsilon$, there is a constant $\alpha$, so that

$$
\frac{1}{\frac{\eta_{i}\left(l_{i} a\right)}{a^{2}}+\mu_{i}^{2}} \leqq \frac{\alpha}{l_{i}^{2}+\mu_{i}^{2}},
$$


whenever $\left\|l_{i}\right\|<(\pi / a) \varepsilon$. This can be seen by an expansion of $\eta_{i}$ at vanishing momentum. Furthermore, there is a constant $\gamma>0$ such that, if $\left\|l_{i}-(2 \pi / a) z\right\|$ $\geqq(\pi / a) \varepsilon$ for all $z \in \mathbf{Z}^{4}$, then

$$
\frac{1}{\frac{\eta_{i}\left(l_{i} a\right)}{a^{2}}+\mu_{i}^{2}} \leqq \gamma a^{2}
$$

Hence, the denominator in every integral (4-6) is bounded by a product of continuum propagators and explicit powers of the lattice spacing $a$. If it would be possible to estimate the numerator $V(k, q ; a)$ by its continuum limit, (4.6) would be bounded by a rational function to which the power counting theorem of Hahn and Zimmermann could be applied (in a somewhat generalized form to determine the cutoff behaviour). Unfortunately, this will not be possible, as we have seen in the introduction. Another possibility would be to expand $V$ at small lattice spacing $a$,

$$
V(k, q ; a)=P(k, q)+R(k, q ; a)
$$

$P$ being the continuum limit of $V$ and $R$ a Taylor remainder, and to estimate $R$ by a polynomial. But this estimate is too rough, the conditions (3.9) will not be sufficient for convergence of this estimate. This is because we have a lot of Zimmermann subspaces and for every such space a corresponding lattice degree of the numerator $V$. For a fixed space we will get an estimate of the form (2.12), but now we need such an inequality which respects degree properties of all Zimmermann subspaces simultaneously. This is not possible in general.

A way out is the following. A simultaneous estimate which respects degree properties can be done for ordered subspace $H_{1}, \ldots, H_{s}$, i.e., $H_{i}$ is a subspace of $H_{j}$ if $i<j$. This means that for every such sequence we get an estimate

$$
|R(u, w ; a)| \leqq a^{p} \sum_{b \in B}\left|Q_{b}(u, w)\right|
$$

where $p$ is a natural number, $B$ a finite set, and the polynomials $Q_{b}$ satisfy

$$
\overline{\operatorname{degr}}_{u_{j}} Q_{b} \leqq \overline{\operatorname{degr}}_{\hat{u}_{j}} V+p, \quad j=1, \ldots, s,
$$

$\left(u_{j}\right)$ being the internal parameters of $H_{j}$. Doing so for every ordered sequence of Zimmermann subspaces, we get

$$
|R(k, q ; a)| \leqq \min _{i \in I} a^{p_{\imath}} \sum_{b \in B_{i}}\left|Q_{i b}(k, q)\right|,
$$

so that for every ordered sequence there exists an $i \in I$ such that

$$
\overline{\operatorname{degr}}_{u} Q_{i b} \leqq \overline{\operatorname{degr}} \hat{u} V+p_{i} \text { for all } b \in B_{i},
$$

$(u)$ being the parameters of an arbitrary Zimmermann subspace in the sequence. Using this and the above estimates for propagators in the integral (4.6), we get generalized continuum Feynman integrals, i.e., integrals which look like Feynman integrals in the continuum with a sharp cutoff, the right-hand side of (4.12) being the numerator. In the next section we will state a theorem which controls the cutoff 
dependence of integrals having this form. Furthermore, we will prove the validity of an inequality (4.12). Using these two statements it will be possible to complete the proof of the power counting theorem under the conditions (3.9).

\section{A Power Counting Theorem for Generalized Continuum Feynman Integrals}

In the present section we state an auxiliary theorem which will be used to complete the proof of the power counting theorem. Set $k=\left(k_{1}, \ldots, k_{m}\right)$ (loop-momenta) and $q$ $=\left(q_{1}, \ldots, q_{M}\right)$ (external momenta), $k_{i}, q_{j} \in \mathbf{R}^{4} . L$ again denotes the space of linear mappings $l: \mathbf{R}^{4 m} \times \mathbf{R}^{4 M} \rightarrow \mathbf{R}^{4}$ of the form (3.2)-(3.4) in the four-momenta $k_{1}, \ldots, k_{m}$ and $q_{1}, \ldots, q_{M}$. The notion of a basis of a set of line momenta is defined in (3.5)-(3.6).

Let $\mathscr{L} \subset L$ be a finite subset

where

$$
\mathscr{L}=\left\{l_{i}(k, q)=\sum_{j=1}^{m} C_{i j} k_{j}+Q_{i}(q) \mid \quad i=1, \ldots, N\right\},
$$

$$
\begin{aligned}
& \operatorname{rank}\left(C_{i j}\right)=m, \\
& \left(C_{i 1}, \ldots, C_{i m}\right) \neq 0 \quad \text { for all } i=1, \ldots, N \text {, } \\
& l_{i}^{2} \neq l_{j}^{2} \quad \text { if } \quad i \neq j,
\end{aligned}
$$

so that $\operatorname{rank}_{k} \mathscr{L}=m$ [cf. (3.6), especially $N \geqq m$ ]. Furthermore, let $\mathscr{N} \subseteq \mathscr{L}$ be an arbitrary subset. We consider integrals of the form

$$
\mathscr{I}_{\lambda}(q, \mu)=\int^{\lambda, \mathscr{L}} d^{4} k_{1} \ldots d^{4} k_{m} \frac{\min _{i \in I} \lambda^{-p_{i}}\left|P_{i}(k, q)\right|}{E(k, q, \mu)}, \quad p_{i} \geqq 0 \text { integer } .
$$

$I$ is a finite set, $P_{i}$ are polynomials in the components of the four-momenta $k_{1}, \ldots, k_{m}$ and $q_{1}, \ldots, q_{M}$, and

$$
E(k, q, \mu)=\prod_{\mathscr{N}}\left(l_{i}^{2}(k, q)+\mu_{i}^{2}\right)^{n_{\mathfrak{\imath}}}, \quad \mu_{i}^{2}>0, \quad n_{i} \in \mathbf{N}=\{1,2, \ldots\} .
$$

$\prod$ means product over $l_{j} \in \mathscr{N}$. Hence $\mathscr{N}$ is the set of all $l_{i} \in \mathscr{L}$ appearing in the I denominator of the integrand of $\mathscr{I}_{\lambda}$. We always have $\operatorname{rank}_{k} \mathscr{N} \leqq m$. All propagators are assumed to be massive.

For a finite subset $\mathscr{M} \subset L$ satisfying $\operatorname{rank}_{k} \mathscr{M}=m$, we define

$$
\int^{\lambda, \mu} d^{4} k_{1} \ldots d^{4} k_{m} f(k)
$$

as the integral over all $\left(k_{1}, \ldots, k_{m}\right) \in \mathbf{R}^{4 m}$ subject to the constraints

$$
l^{2}(k, q) \leqq \lambda^{2} \text { for all } l \in \mathscr{M} .
$$

$\mathscr{I}_{\lambda}$ is convergent for every finite $\lambda$. We examine the behaviour of $\mathscr{I}_{\lambda}$ for large $\lambda$. The cutoff dependence can be described with the help of divergence degrees with respect to Zimmermann subspaces of the integration momenta. First we make the notions 
more precise. Let

$$
\begin{gathered}
u_{1}=l_{i_{1}}, \ldots, u_{d}=l_{i_{d}}, \\
v_{1}=l_{j_{1}}, \ldots, v_{m-d}=l_{j_{m-d}}
\end{gathered}
$$

be an arbitrary basis of $\mathscr{L}$ with respect to $k$, so that $k=k(u, v, q), 1 \leqq d \leqq m$. As in Sect. 3 , by fixing $v_{1}, \ldots, v_{m-d}$, we define a class $H$ of affine subspaces in the space of integration momenta $k$ called Zimmermann subspace. $(u)=\left(u_{1}, \ldots, u_{d}\right)$ is called the parametrization of $H .\left(v_{1}, \ldots, v_{m-d}\right)$ are said to be the complementary parameters of $H$. The set of all $H$, for all bases (5.6) of $\mathscr{L}$, is denoted by $\mathscr{H} . \mathscr{H}$ is the set of all possible Zimmermann subspaces, and it depends on the set $\mathscr{L}$.

Definition 5.1. Let

$$
u^{(1)}, \ldots, u^{(m)}
$$

be an arbitrary basis of $\mathscr{L}$ and $H_{1}, \ldots, H_{s}, s \geqq 1$, a sequence of classes of affine subspaces in $\mathscr{H}$ having the following properties:

1. $H_{i}$ is parametrized by $\left(u_{i}\right)=\left(u_{i 1}, \ldots, u_{i d_{z}}\right) \subseteq\left\{u^{(1)}, \ldots, u^{(m)}\right\}$, the remaining $u^{(j)}$ 's in the basis being the complementary parameters of $H_{i}$.

2. $\left(u_{j}\right)$ are contained in $\left(u_{k}\right)$ if $j<k$.

Then the sequence $H_{1}, \ldots, H_{s}$ is called an ordered sequence with respect to the basis (5.7a).

With respect to the set of polynomials $\left\{P_{i} \mid i \in I\right\}$ in the numerator of (5.2) we define

Definition 5.2. The set $\{\delta(H) \mid H \in \mathscr{H}\}$ is called an ultraviolet-set (UV-set), if

1. $\delta(H) \in \mathbf{Z}$ for every $H \in \mathscr{H}$.

2. For every basis $\left(u^{(1)}, \ldots, u^{(m)}\right)$ of $\mathscr{L}$ and every sequence $H_{1}, \ldots, H_{s}$ which is ordered with respect to this basis, there exists an $i \in I$ such that (cf. Appendix C)

$$
\overline{\operatorname{degr}}_{u_{j} \mid v_{j}} P_{i}(k, q)-p_{i} \leqq \delta\left(H_{j}\right) \text { for all } j=1, \ldots, s \text {. }
$$

Here $\left(u_{j}\right)$ denotes the parameters of $H_{j}$ and $\left(u_{j}, v_{j}\right)=\left(u^{(1)}, \ldots, u^{(m)}\right)$.

The number of possible bases of $\mathscr{L}$ and ordered sequences of subspaces in $\mathscr{H}$ is finite. Hence UV-sets do always exist. If $I=\{1\}$ consists of one element only, the set of

$$
\delta(H)=\overline{\operatorname{degr}}_{u \mid v} P_{1}(k(u, v, q), q)-p_{1}
$$

for every $H \in \mathscr{H}$, where $(u)$ is the parametrization of $H$ and $(v)$ are the complementary variables, is a UV-set. In this case the notion of ordered subspaces is superfluous. Note that UV-sets as defined in (5.8) are dependent on the external momenta $q$, which we have kept fixed. However, for every UV-set $\{\delta(H) \mid H \in \mathscr{H}\}$ one can find a UV-set $\left\{\delta^{\prime}(H) \mid H \in \mathscr{H}\right\}$, which is independent of $q$, where $\delta^{\prime}(H) \geqq \delta(H)$ for all $H \in \mathscr{H}$.

We now define UV-divergence degrees for integrals of the form (5.2). UVdegrees of polynomials are defined in Appendix C. Let $\{\delta(H) \mid H \in \mathscr{H}\}$ be a UV-set. 
Given an arbitrary basis (5.6) of $\mathscr{L}$, we define for $H \in \mathscr{H}$, parametrized by $(u)=\left(u_{1}, \ldots, u_{d}\right)$,

$$
\omega(H)=\overline{\operatorname{degr}}_{H} \mathscr{I}_{\lambda} \equiv 4 d+\delta(H)-\overline{\operatorname{degr}}_{u \mid v} E(k(u, v, q), q, \mu) .
$$

This definition depends on a given UV-set. The following theorem states the cutoff behavior of integrals (5.2) for large $\lambda$ if a UV-set is given.

Theorem 2. Auxiliary Power Counting Theorem. Let $\{\delta(H) \mid H \in \mathscr{H}\}$ be a UV-set and $\{\omega(H) \mid H \in \mathscr{H}\}$ the corresponding set of UV-divergence degrees. Then there exist $K(\mu, q)>0$ and $c(\mu, q)>0$, so that for all $\lambda>K(\mu, q)$,

$$
\mathscr{I}_{\lambda}(q, \mu) \leqq c(\mu, q) \cdot \begin{cases}1 & \text { if } \max _{H \in \mathscr{H}} \omega(H)<0 \\ \lambda^{-1} \log ^{m} \lambda & \text { if } \max _{H \in \mathscr{H}} \omega(H)<0 \text { and } p_{i} \geqq 1 \text { for all } i \in I \\ \lambda^{\max \omega(H)} \log ^{m} \lambda & \text { if } \max _{H \in \mathscr{H}} \omega(H) \geqq 0 .\end{cases}
$$

If the momenta q are bounded and the UV-set is independent of such q, then Kand c can be chosen to be independent of $q$.

The estimate (5.10) can be strenghened if a UV-set is given having $\max \omega(H)$ minimal. However, we do not need this in our application, where a UV-set will be given in a natural way. The theorem is an extension of the power counting theorem of Hahn and Zimmermann [1]. In general, the numerator is not a polynomial, instead it is a minimum of a collection of polynomials, and we include the cutoff behavior of divergent integrals (for $\lambda \rightarrow \infty$ ). Below we will apply the theorem (for $\lambda$ $\sim 1 / a)$ in two special cases. If $I=\{1\}$ and $p_{1}=0$, the statement of [1] is reproduced. If all $p_{i} \geqq 1$ and the limit exists, it is zero. If $p_{i}=0$ for all $i \in I$ and $\max _{H \in \mathscr{H}} \omega(H)<0$, then $\mathscr{I}_{\lambda}(q, \mu)$ converges to

$$
\int_{-\infty}^{\infty} d^{4} k_{1} \ldots d^{4} k_{m} \frac{\min _{i \in I}\left|P_{i}(k, q)\right|}{E(k, q, \mu)} .
$$

The proof of Theorem 2 will be given in Sect. 8,9.

\section{Bounds on the Numerator of a Lattice Feynman Integrand}

Having introduced appropriate notions and an auxiliary power counting theorem being at our disposal, we will now show that the numerator of a Feynman integrand can be estimated as proposed at the end of Sect. 4. This statement is contained in the following theorem. It is a consequence of the definition of UV-degrees on the lattice. Remember we are using multi-index notation. We shall write $k=\left(k_{1}, \ldots, k_{m}\right)$ and $q$ $=\left(q_{1}, \ldots, q_{M}\right)$ as in the power counting theorem. 
Theorem 3. Let $V(k, q ; a) \in \mathscr{C}_{m_{0}}^{\mathrm{c}}$ for some $m_{0} \in \mathbf{Z}, \mathscr{L}$ a natural set of four-vectors with respect to $k^{4}$, and let $(k a, q a)$ be bounded. Then $V$ admits an estimate of the form

$$
|V(k, q ; a)-P(k, q)| \leqq a^{p} \sum_{b \in B} \min _{i \in I}\left|Q_{i b}(k, q)\right|
$$

where $I, B$ are finite sets, $p \in \mathbf{N}$, and

1. $P(k, q)=\lim _{a \rightarrow 0} V(k, q ; a)$. For every $H \in \mathscr{H}$, parametrized by $(u)$

$$
\overline{\operatorname{degr}_{u}} P \leqq \overline{\operatorname{degr}_{\hat{u}}} V .
$$

2. $Q_{i b}$ are polynomials. For every basis

$$
u^{(1)}=l_{i_{1}}, \ldots, u^{(m)}=l_{i_{m}}
$$

of $\mathscr{L}$ with respect to $k$ and every sequence $H_{1}, \ldots, H_{s}$ of classes of affine subspaces in $\mathscr{H}$ which is ordered with respect to the basis (6.3), there exists an $i \in I$, so that

$$
\overline{\operatorname{degr}}_{u_{j}} Q_{i b} \leqq \overline{\operatorname{degr}}_{\hat{u}_{j}} V+p \quad \text { for all } j=1, \ldots, s \text { and all } b \in B,
$$

where $\left(u_{j}\right)$ denotes the set of parameters of $H_{j}$.

The statement means that, for every $b \in B$, the set of all $\delta(H)=\overline{\operatorname{degr}}_{\hat{u}} V$, where $(u)$ is the parametrization of $H \in \mathscr{H}$, is a UV-set for the polynomials $Q_{i b}$, which is independent of $q$. This allows us to apply the cutoff theorem to the integrals (4.6) in Sect. 4 to determine their cutoff dependence, as will be seen in the next section. Note that always $p \geqq 1$. If $P(k, q) \neq 0$, then $p$ can be chosen to be 1 . If $V(k, q ; a)$ is the numerator of a Feynman integral, the variables $(k a, q a)$ are always bounded, because $k_{1}, \ldots, k_{m}$ range over the $\mathrm{BZ}$, and external momenta $q$ are fixed.

In the remaining part of this section Theorem 3 is proved. First of all we note an extended version of Taylor's theorem.

Lemma 6.1. Let $F$ be a $C^{\infty}$-function of the form $F\left(v_{1}, \ldots, v_{n}\right), v_{i} \in \mathbf{R}^{m_{\imath}}$. Let $\delta_{i} \in \mathbf{N}_{0}$ $=\{0,1,2, \ldots\}$ for every $i=1, \ldots, n$, and $\delta_{i} \geqq \delta_{k}$ if $i<k$. If

$$
F\left(v_{1}, \ldots, v_{s-1}, \lambda v_{s}, \ldots, \lambda v_{n}\right)=O\left(\lambda^{\delta_{s}}\right), \quad \lambda \rightarrow 0 ; s=1, \ldots, n,
$$

then there exist $C^{\infty}$-functions $F_{b}, b \in \mathbf{N}_{0}^{m_{n}},|b|=\delta_{n}$, satisfying

$$
F_{b}\left(v_{1}, \ldots, v_{s-1}, \lambda v_{s}, \ldots, \lambda v_{n}\right)=O\left(\lambda^{\delta_{s}-\delta_{n}}\right), \quad \lambda \rightarrow 0
$$

for all $s=1, \ldots, n-1$, so that

$$
F\left(v_{1}, \ldots, v_{n}\right)=\sum_{b} v_{n}^{b} F_{b}\left(v_{1}, \ldots, v_{n}\right) .
$$

This lemma is an extension of Taylor's formula in the sense that it states the coefficient functions $F_{b}$ being $C^{\infty}$ if this already holds for $F$. This allows successive application of (6.7).

\footnotetext{
${ }^{4}$ It would be sufficient to assume property 1 in the definition of naturalness (existence of a basis). However, in application of Theorem 3 below, $\mathscr{L}$ will be natural
} 
Proof. By induction on $m_{n}$. If $m_{n}=1$, let $b=\delta_{n} \in \mathbf{N}_{0}$ and

$$
F_{b}\left(v_{1}, \ldots, v_{n}\right)=\frac{F\left(v_{1}, \ldots, v_{n}\right)}{v_{n}^{b}} .
$$

By Taylor's formula, $F_{b} \in C^{\infty}$, and (6.6) is satisfied. Assume the statement holds for all $m_{n} \leqq M$, where $M \in \mathbf{N}$. Let $v_{n}=\left(w_{1}, w_{2}\right), w_{1} \in \mathbf{R}^{M}, w_{2} \in \mathbf{R}$. For $l=0, \ldots, \delta_{n}-1$ set

$$
A_{l}\left(v_{1}, \ldots, v_{n-1}, w_{1}\right)=\left[\frac{\partial^{l}}{\partial w_{2}^{l}} F\left(v_{1}, \ldots, v_{n-1}, w_{1}, w_{2}\right)\right]_{w_{2}=0},
$$

and define a function $G$ by

$$
G\left(v_{1}, \ldots, v_{n-1}, w_{1}, w_{2}\right)=F\left(v_{1}, \ldots, v_{n-1}, w_{1}, w_{2}\right)-\sum_{l=0}^{\delta_{n}-1} \frac{w_{2}^{l}}{l !} A_{l}\left(v_{1}, \ldots, v_{n-1}, w_{1}\right)
$$

The hypothesis of induction can be applied to $G$ and $A_{l}$. For, as $\lambda \rightarrow 0$ we have

$$
\begin{aligned}
A_{l}\left(v_{1}, \ldots, v_{n-1}, \lambda w_{1}\right) & =O\left(\lambda^{\delta_{n}-l}\right), \\
A_{l}\left(v_{1}, \ldots, v_{s-1}, \lambda v_{s}, \ldots, \lambda v_{n-1}, \lambda w_{1}\right) & =O\left(\lambda^{\delta_{s}-l}\right) ; \quad 1 \leqq s \leqq n-1, \\
G\left(v_{1}, \ldots, v_{n-1}, w_{1}, \lambda w_{2}\right) & =O\left(\lambda^{\delta_{n}}\right), \\
G\left(v_{1}, \ldots, v_{s-1}, \lambda v_{s}, \ldots, \lambda v_{n-1}, \lambda w_{1}, \lambda w_{2}\right) & =O\left(\lambda^{\delta_{s}}\right) ; \quad 1 \leqq s \leqq n-1 .
\end{aligned}
$$

Hence, there exist $g\left(v_{1}, \ldots, v_{n-1}, w_{1}, w_{2}\right) \in C^{\infty}$ and $h_{l, b}\left(v_{1}, \ldots, v_{n-1}, w_{1}\right) \in C^{\infty}$ for all $b \in \mathbf{N}_{0}^{M},|b|=\delta_{n}-l$, so that

$$
A_{l}\left(v_{1}, \ldots, v_{n-1}, w_{1}\right)=\sum_{b} w_{1}^{b} h_{l, b}\left(v_{1}, \ldots, v_{n-1}, w_{1}\right)
$$

$h_{l, b}\left(v_{1}, \ldots, v_{s-1}, \lambda v_{s}, \ldots, \lambda v_{n-1}, \lambda w_{1}\right)=O\left(\lambda^{\delta_{s}-\delta_{n}}\right) ; \quad \lambda \rightarrow 0 \quad ; \quad 1 \leqq s \leqq n-1$,

and

$$
\begin{gathered}
G\left(v_{1}, \ldots, v_{n-1}, w_{1}, w_{2}\right)=w_{2}^{\delta_{n}} g\left(v_{1}, \ldots, v_{n-1}, w_{1}, w_{2}\right) \\
g\left(v_{1}, \ldots, v_{s-1}, \lambda v_{s}, \ldots, \lambda v_{n-1}, \lambda w_{1}, \lambda w_{2}\right)=O\left(\lambda^{\delta_{s}-\delta_{n}}\right), \quad \lambda \rightarrow 0 ; \quad 1 \leqq s \leqq n-1 .
\end{gathered}
$$

Writing $F$ in terms of $G$ and $A_{l}$, the assertion follows.

We shall use the following notation: For $s \in \mathbf{N}, \delta=\left(\delta_{s}, \ldots, \delta_{n}\right) \in \mathbf{N}_{0}^{n-s+1}$ and multi-indices $b_{i} \in \mathbf{N}_{0}^{m_{l}}, i=s, \ldots, n$ let

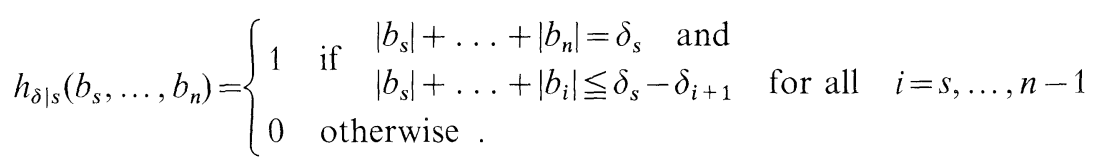

By iteration of Lemma 6.1 we get

Lemma 6.2. Let $F$ be a $C^{\infty}$-function of the form $F\left(v_{1}, \ldots, v_{n}\right), v_{i} \in \mathbf{R}^{m_{2}}$, and $\delta_{i} \in \mathbf{N}_{0}$ for every $i=1, \ldots, n, \delta_{i} \geqq \delta_{k}$ if $i<k$. If as $\lambda \rightarrow 0$

$$
F\left(v_{1}, \ldots, v_{t-1}, \lambda v_{t}, \ldots, \lambda v_{n}\right)=O\left(\lambda^{\delta_{t}}\right), \quad t=1, \ldots, n,
$$


then for arbitrary $s=1, \ldots, n$ there exist functions $F_{b_{s} \ldots b_{n}} \in C^{\infty}$, so that

$$
\begin{gathered}
F\left(v_{1}, \ldots, v_{n}\right)=\sum_{b_{s} \ldots b_{n}} h_{\delta \mid s}\left(b_{s}, \ldots, b_{n}\right) v_{s}^{b_{s}} \ldots v_{n}^{b_{n}} F_{b_{s} \ldots b_{n}}\left(v_{1}, \ldots, v_{n}\right), \\
F_{b_{s} \ldots b_{n}}\left(v_{1}, \ldots, v_{t-1}, \lambda v_{t}, \ldots, \lambda v_{n}\right)=O\left(\lambda^{\delta_{t}-\delta_{s}}\right), \quad \lambda \rightarrow 0 ; \quad 1 \leqq t<s .
\end{gathered}
$$

Proof. If $s=n$, this is the statement of Lemma 6.1. Assume it holds for some $s \geqq 2$. Application of Lemma 6.1 to $F_{b_{s} \ldots b_{n}}$ in (6.9) yields

$$
\begin{gathered}
F_{b_{s} \ldots b_{n}}\left(v_{1}, \ldots, v_{n}\right)=\sum_{\left|c_{s-1}\right|+\ldots+\left|c_{n}\right|=\delta_{s-1}-\delta_{s}} v_{s-1}^{c_{s-1}} \ldots v_{n}^{c_{n}} F_{b_{s} \ldots b_{n}, c_{s-1} \ldots c_{n}}\left(v_{1}, \ldots, v_{n}\right), \\
F_{b_{s} \ldots b_{n}, c_{s-1} \ldots c_{n}}\left(v_{1}, \ldots, v_{t-1}, \lambda v_{t}, \ldots, \lambda v_{n}\right)=O\left(\lambda^{\delta_{t}-\delta_{s-1}}\right), \quad \lambda \rightarrow 0 ; \quad 1 \leqq t<s-1 .
\end{gathered}
$$

Inserting this into (6.9) and collecting indices of $F_{b_{s} \ldots b_{n}, c_{s-1} \ldots c_{n}}$, we obtain

$$
\begin{gathered}
F\left(v_{1}, \ldots, v_{n}\right)=\sum_{b_{s-1} \ldots b_{n}} h_{\delta \mid s-1}\left(b_{s-1}, \ldots, b_{n}\right) v_{s-1}^{b_{s}-1} \ldots v_{n}^{b_{n}} F_{b_{s-1} \ldots b_{n}}\left(v_{1}, \ldots, v_{n}\right), \\
F_{b_{s-1} \ldots b_{n}}\left(v_{1}, \ldots, v_{t-1}, \lambda v_{t}, \ldots, \lambda v_{n}\right)=O\left(\lambda^{\delta_{t}-\delta_{s-1}}\right), \quad \lambda \rightarrow 0 ; \quad 1 \leqq t<s-1 .
\end{gathered}
$$

Lemma 6.3. Let

$$
u^{(1)}=l_{i_{1}}, \ldots, u^{(m)}=l_{i_{m}}
$$

be an arbitrary basis of $\mathscr{L}$ with respect to $k$, and $H_{1}, \ldots, H_{s}$ an arbitrary sequence of Zimmermann subspaces which is ordered with respect to (6.11). Furthermore, let $V(k, q ; a) \in \mathscr{C}_{m_{0}}^{c}, m_{0} \in \mathbf{Z}$, and $(k a, q a)$ be bounded. Then $V$ admits an estimate of the form

$$
|V(k, q ; a)-P(k, q)| \leqq a^{p} \sum_{b \in B}\left|Q_{b}(k, q)\right|,
$$

where $B$ is a finite set. $p \in \mathbf{N}$ is independent of the basis (6.11) and the sequence of subspaces. The homogeneous polynomial $P$ is given by $P(k, q)=\lim V(k, q ; a)$, and $Q_{b}$ are homogeneous polynomials of order $p+m_{0}$ such that

$$
\begin{aligned}
& \overline{\operatorname{degr}}_{u_{j}} P \leqq \overline{\operatorname{degr}}_{\hat{u}_{j}} V, \\
& \overline{\operatorname{degr}}_{u_{j}} Q_{b} \leqq p+\overline{\operatorname{degr}}_{\hat{u}_{J}} V,
\end{aligned}
$$

where $\left(u_{j}\right)$ is the parametrization of $H_{j}$, for all $j=1, \ldots, s$ and all $b \in B$.

If $P(k, q) \neq 0, p$ can be chosen to be 1 . If $P(k, q) \equiv 0, p$ is the largest natural number such that $\lim _{a \rightarrow 0} V(k, q ; a) / a^{p} \neq 0$ exists.

Proof. 1. We define new sets of variables $v_{1}, \ldots, v_{s+1}$ as follows:

$$
\begin{aligned}
\left(u_{1}\right) & =\left(v_{1}\right), \\
\left(u_{2}\right) & =\left(v_{1}, v_{2}\right), \\
& \vdots \\
\left(u_{s}\right) & =\left(v_{1}, \ldots, v_{s}\right), \\
\left(u^{(1)}, \ldots, u^{(m)}, q\right) & =\left(v_{1}, \ldots, v_{s+1}\right) \equiv(v) .
\end{aligned}
$$


Let $V(k, q ; a)=F(k a, q a) / a^{m_{0}} \in \mathscr{C}_{m_{0}}^{c}$ and $F^{\prime}(v)=F(k, q)$. For every $H_{j}$ in the given ordered sequence we make a partition $(v)=\left(u_{j}, w_{j}\right)$, where $\left(u_{j}\right)=\left(v_{1}, \ldots, v_{j}\right)$ are the "internal" momenta and $\left(w_{j}\right)=\left(v_{j+1}, \ldots, v_{s+1}\right)$ are the "external" momenta of $H_{j}$. Set $r_{j}=m_{0}-\overline{\operatorname{degr}} \hat{u}_{j} V$. Then $r_{1} \geqq \ldots \geqq r_{s}$ and

$$
F^{\prime}\left(v_{1}, \ldots, v_{j}, \lambda v_{j+1}, \ldots, \lambda v_{s+1}\right)=O\left(\lambda^{r_{j}}\right), \quad \lambda \rightarrow 0 ; j=1, \ldots, s .
$$

2. For $\delta \in \mathbf{Z}$ define

and set

$$
P_{\delta}^{\prime}\left(v_{1}, \ldots, v_{s+1}\right)=\lim _{\lambda \rightarrow 0} \frac{F^{\prime}\left(\lambda v_{1}, \ldots, \lambda v_{s+1}\right)}{\lambda^{\delta}}
$$

$$
G\left(v_{1}, \ldots, v_{s+1}\right)=F^{\prime}\left(v_{1}, \ldots, v_{s+1}\right)-P_{m_{0}}^{\prime}\left(v_{1}, \ldots, v_{s+1}\right) .
$$

Let $r_{0} \in \mathbf{N}_{0}$ be the largest integer number such that $P_{r_{0}}^{\prime}\left(v_{1}, \ldots, v_{s+1}\right) \neq 0$ exists. Set

$$
\hat{r}_{0}=\left\{\begin{array}{lll}
r_{0} & \text { if } & m_{0}<r_{0} \\
r_{0}+1 & \text { if } & m_{0}=r_{0}
\end{array}\right.
$$

and $\hat{r}_{j}=r_{j}$ for $j \geqq 1$. Then

$$
G\left(v_{1}, \ldots, v_{j}, \lambda v_{j+1}, \ldots, \lambda v_{s+1}\right)=O\left(\lambda^{\hat{r}_{j}}\right) ; \quad \lambda \rightarrow 0 \quad, \quad 0 \leqq j \leqq s,
$$

and $\hat{r}_{0} \geqq \hat{r}_{1} \geqq \ldots \geqq \hat{r}_{s}$. Applying Lemma 6.2 to $G$ yields

$$
G\left(v_{1}, \ldots, v_{s+1}\right)=\sum_{b_{1}, \ldots, b_{s+1}} h_{\mathbf{r}\rceil 1}\left(b_{1}, \ldots, b_{s+1}\right) v_{1}^{b_{1}} \ldots v_{s+1}^{b_{s+1}} F_{b_{1} \ldots b_{s+1}}\left(v_{1}, \ldots, v_{s+1}\right)
$$

where

$$
h_{\hat{r} \mid 1}\left(b_{1}, \ldots, b_{s+1}\right)= \begin{cases}1 & \text { if } \quad\left|b_{1}\right|+\ldots+\left|b_{s+1}\right|=\hat{r}_{0} \text { and } \\ & \left|b_{1}\right|+\ldots+\left|b_{j}\right| \leqq \hat{r}_{0}-\hat{r}_{j} \text { for all } j=1, \ldots, s \\ 0 & \text { otherwise },\end{cases}
$$

and $F_{b_{1} \ldots b_{s+1}} \in C^{\infty}$. For bounded $v a$ we get

$$
\left|G\left(v_{1} a, \ldots, v_{s+1} a\right)\right| \leqq a^{\hat{r}_{0}} \sum_{b \in B}\left|Q_{b}^{\prime}\left(v_{1}, \ldots, v_{s+1}\right)\right|,
$$

where $Q_{b}^{\prime}$ are (finitely many) homogeneous polynomials of order $\hat{r}_{0}$ satisfying

$$
\overline{\operatorname{degr}}_{v_{1} \ldots v_{j}} Q_{b}^{\prime} \leqq \hat{r}_{0}-\hat{r}_{j}=\left(\hat{r}_{0}-m_{0}\right)+\overline{\operatorname{degr}}_{\hat{u}_{j}} V, \quad j=1, \ldots, s,
$$

for all $b \in B$. Finally, let

$$
\begin{gathered}
P(k, q) \equiv \lim _{a \rightarrow 0} \frac{1}{a^{m_{0}}} V(k, q ; a) \equiv P_{m_{0}}^{\prime}\left(v_{1}, \ldots, v_{s+1}\right), \\
Q_{b}(k, q) \equiv Q_{b}^{\prime}\left(v_{1}, \ldots, v_{s+1}\right) \text { for all } b \in B .
\end{gathered}
$$

Setting $p=\hat{r}_{0}-m_{0} \in \mathbf{N}$, (6.13) follows, and as a consequence of (6.14)

$$
|V(k, q ; a)-P(k, q)|=\frac{1}{a^{m_{0}}}\left|G\left(v_{1} a, \ldots, v_{s+1} a\right)\right| \leqq a^{p} \sum_{l \in L}\left|Q_{l}(k, q)\right| .
$$


Proof of Theorem 3. Let the set of all ordered sequences of subspaces in $\mathscr{H}$ be indexed by a finite set $I$. Using Lemma $6.3, V \in \mathscr{C}_{m_{0}}^{c}$ admits for every $i \in I$ an estimate of the form

$$
|V(k, q ; a)-P(k, q)| \leqq a^{p} \sum_{b \in B_{\imath}}\left|Q_{b}(k, q)\right|,
$$

where $B_{i}$ is a finite set and $p \in \mathbf{N}$ is independent of $i \in I$. $P$ is equal to the continuum limit of $V$, hence is independent of all sequences, and satisfies for every $H \in \mathscr{H}$, parametrized by $(u)$,

$$
\overline{\operatorname{degr}}_{u} P \leqq \overline{\operatorname{degr}}_{\hat{u}} V .
$$

For every $H_{j}$, parametrized by $\left(u_{j}\right)$, in the ordered sequence,

$$
\overline{\operatorname{degr}}_{u_{j}} Q_{b} \leqq p+\overline{\operatorname{degr}}_{\hat{u}_{j}} V, \quad \text { for all } b \in B_{i} .
$$

In summary, using the inequality and notations of Appendix B, $V$ obeys an inequality

$$
|V(k, q ; a)-P(k, q)| \leqq a^{p} \min _{i \in I} \sum_{b \in B_{i}}\left|Q_{b}(k, q)\right| \leqq a^{p} \sum_{b \in B} \min _{i \in I}\left|Q_{i b}(k, q)\right|,
$$

where $B=\otimes_{i \in I}^{\otimes} B_{i}$, and for $b=\left(b_{i}\right)_{i \in I} \in B, Q_{i b}=Q_{b_{i}}$. Point 2 in Theorem 3 is satisfied by construction.

\section{Completion of the Proof of the Power Counting Theorem}

Having shown that the numerator of a lattice Feynman integrand admits an estimate as supposed at the end of Sect. 4, and a theorem being at our disposal which states the cutoff dependence of generalized continuum Feynman integrals, it is not hard to complete the proof of Theorem 1. Our starting point is (4.6). Using Theorem 3 we write $V(k, q ; a)=P(k, q)+R(k, q ; a)$, so that

where

$$
\hat{I}_{J z}=\hat{I}_{J z}^{0}+\hat{I}_{J z}^{R}
$$

$$
\hat{I}_{J z}^{0}=\int_{\sigma_{J}} d^{4} k_{1} \ldots d^{4} k_{m} \frac{P(k, q)}{\prod_{i=1}^{n}\left(\frac{\eta_{i}\left(l_{i} a\right)}{a^{2}}+\mu_{i}^{2}\right)}\left(\prod_{i \in J} \Theta\left(\frac{\pi}{a} \varepsilon-\left\|l_{i}\right\|\right)\right) \cdot \prod_{i \notin J} \Theta_{\varepsilon}\left(l_{i}\right),
$$

and

$$
\hat{I}_{J z}^{R}=\int_{\sigma_{J}} d^{4} k_{1} \ldots d^{4} k_{m} \frac{R(k, q ; a)}{\prod_{i=1}^{n}\left(\frac{\eta_{i}\left(l_{i} a\right)}{a^{2}}+\mu_{i}^{2}\right)}\left(\prod_{i \in J} \Theta\left(\frac{\pi}{a} \varepsilon-\left\|l_{i}\right\|\right)\right) \cdot \prod_{i \notin J} \Theta_{\varepsilon}\left(l_{i}\right) .
$$

$P$ is the continuum limit of $V$, and $R(k, q ; a)$ admits an estimate

$$
|R(k, q ; a)| \leqq a^{p} \sum_{b \in B} \min _{i \in I}\left|Q_{i b}(k, q)\right|
$$


By Theorem 3, for every fixed $b \in B$, the set of all $\delta(H)=\overline{\operatorname{degr}}_{\hat{u}} V,(u)$ being the parametrization of $H \in \mathscr{H}$, is a UV-set for the polynomials $Q_{i b}$.

Using the bounds (4.8), (4.9) on the propagators, we get the estimates

$$
\left|\hat{I}_{J z}^{0}(q ; \mu, a)\right| \leqq \bar{I}_{J z}^{0}(q, \mu, a)=\alpha^{h}\left(\gamma a^{2}\right)^{n-h} \int_{\kappa_{J}} d^{4} k_{1} \ldots d^{4} k_{m} \frac{|P(k, q)|}{\prod_{i \in J}\left(l_{i}^{2}+\mu_{i}^{2}\right)},
$$

and

where

$$
\left|\hat{I}_{J z}^{R}(q ; \mu, a)\right| \leqq \sum_{b \in B} \bar{I}_{J z}^{(b)}(q, \mu, a)
$$

$$
\bar{I}_{J z}^{(b)}(q, \mu, a)=\alpha^{h}\left(\gamma a^{2}\right)^{n-h} \int_{\kappa_{J}} d^{4} k_{1} \ldots d^{4} k_{m} \frac{\min _{i \in I} a^{p}\left|Q_{i b}(k, q)\right|}{\prod_{i \in J}\left(l_{i}^{2}+\mu_{i}^{2}\right)} .
$$

$h$ is the number of elements of $J$, i.e., it is the number of propagators having a momentum near a pole,

and

$$
\begin{gathered}
\kappa_{J}=\left\{\left(k_{1}, \ldots, k_{m}\right) \in \mathbf{R}^{4 m} \mid\left\|l_{j}\right\| \leqq \delta / a \text { for all } l_{j} \in \mathscr{L}_{J}\right\}, \\
\mathscr{L}_{J}=\left\{l_{j} \mid j \in J\right\} \cup\left\{k_{1}, \ldots, k_{m}\right\} \subseteq \mathscr{L},
\end{gathered}
$$

$$
\delta=\max _{i=1, \ldots, m}\left(\pi \varepsilon, 4 \pi\left(1+\left\|\delta_{i}\right\| / 2\right)\right)
$$

is a constant. To every integral in (7.4) and (7.5) we can now apply the auxiliary power counting theorem to discuss the small $a$ behavior. All integrals are of the form needed, where $\lambda$ is replaced by $\delta / a$ and $\mathscr{L}$ by $\mathscr{L}_{J}$. The corresponding set $\mathscr{H}_{J}$ of Zimmermann subspaces of $k$ is defined by bases of $\mathscr{L}_{J}$ with respect to $k$. By (7.7) $\mathscr{H}_{J} \subseteq \mathscr{H}$. Hence for every $J \subseteq\{1, \ldots, n\}$ the set of $\delta(H)=\overline{\operatorname{degr}}_{\hat{u}} V,(u)$ being the parametrization of $H$, for all $H \in \mathscr{H}_{J}$ is a UV-set for the family of polynomials $Q_{i b}$, for every fixed $b \in B$. It is independent of the external momenta $q$.

We first consider the integrals $\bar{I}_{J z}^{(b)}$. As a consequence of (3.9), for arbitrary $H \in \mathscr{H}_{J}$, parametrized by $(u)=\left(u_{1}, \ldots, u_{d}\right)$ say, we get

$$
\begin{aligned}
\overline{\operatorname{degr}}_{H} \bar{I}_{J z}^{(b)}(q, \mu, a) & \equiv 4 d+\delta(H)-\overline{\operatorname{degr}}_{u} \prod_{i \in J}\left(l_{i}^{2}+\mu_{i}^{2}\right) \\
& =\left[4 d+\overline{\operatorname{degr}}_{\hat{u}} V-\overline{\operatorname{degr}}_{\hat{u}} C\right]+\overline{\operatorname{degr}}_{u} \prod_{i \notin J}\left(l_{i}^{2}+\mu_{i}^{2}\right) \\
& <\overline{\operatorname{degr}}_{u} \prod_{i \notin J}\left(l_{i}^{2}+\mu_{i}^{2}\right) \leqq 2(n-h),
\end{aligned}
$$

where we have used (2.15). Hence

$$
\overline{\operatorname{degr}}_{H} \bar{I}_{J z}^{(b)}(q, \mu, a) \leqq 2(n-h)-1 \text { for all } H \in \mathscr{H}_{J} .
$$

By the auxiliary power counting theorem, there exist positive constants $K$ and $c$, so that for all $a<K^{-1}$,
a) if $n-h>0, \quad \bar{I}_{J z}^{(b)}(q, \mu, a) \leqq c\left(a^{2}\right)^{n-h} a^{-[2(n-h)-1]} \log ^{m} a=c a \log ^{m} a$.
b) if $n-h=0, \quad \bar{I}_{J z}^{(b)}(q, \mu, a) \leqq c a \log ^{m} a \quad$ (because of $p \geqq 1$ ). 
Thus, the remainder $\hat{I}_{J z}^{R}$ does not contribute in the continuum limit.

Next, we turn to the integrals $\bar{I}_{J z}^{0}$. If $P(k, q) \equiv 0$, all $\bar{I}_{J z}^{0}$ vanish and the proof of the power counting theorem is complete. Thus, let us assume that $P(k, q) \neq 0$. For every $H \in \mathscr{H}_{J}$, parametrized by $(u)=\left(u_{1}, \ldots, u_{d}\right)$,

$$
\overline{\operatorname{degr}}_{u} P \leqq \overline{\operatorname{degr}}_{\hat{u}} V \text {. }
$$

The set of $\delta(H)=\overline{\operatorname{degr}}_{u} P(k(u, v, q), q), H \in \mathscr{H}_{J}$, is a UV-set. Consequently, using (7.13) and (3.9), we have

$$
\begin{aligned}
\overline{\operatorname{degr}}_{H} \bar{I}_{J z}^{0}(q, \mu, a) & \equiv 4 d+\overline{\operatorname{degr}}_{u} P(k, q)-\overline{\operatorname{degr}}_{u} \prod_{i \in J}\left(l_{i}^{2}+\mu_{i}^{2}\right) \\
& \leqq\left[4 d+\overline{\operatorname{degr}} \hat{u} V-\overline{\operatorname{degr}}_{\hat{u}} C\right]+\overline{\operatorname{degr}}_{u} \prod_{i \notin J}\left(l_{i}^{2}+\mu_{i}^{2}\right)<2(n-h),
\end{aligned}
$$

and hence

$$
\overline{\operatorname{degr}}_{H} \bar{I}_{J z}^{0}(q, \mu, a) \leqq 2(n-h)-1,
$$

for every $H \in \mathscr{H}_{J}$. Using again the auxiliary power counting theorem, there exist $K>0$ and $c>0$, so that for all $a<K^{-4}$,

$$
\begin{aligned}
& \text { a) if } n-h>0, \quad \bar{I}_{J z}^{0}(q, \mu, a) \leqq c\left(a^{2}\right)^{n-h} \cdot a^{-[2(n-h)-1]} \log ^{m} a=c a \log ^{m} a, \\
& \text { b) if } n-h=0, \quad \bar{I}_{J z}^{0}(q, \mu, a) \leqq c .
\end{aligned}
$$

This shows that in the continuum limit only sectors $\left(J_{0}, z\right)$ where $J_{0}=\{1, \ldots, n\}$, contribute to (4.3), i.e., when the momenta of all propagators are located near the poles. In fact, for appropriate small $\varepsilon>0$, there is exactly one such sector. For, if $P \neq 0$, the set $\left\{l_{1}, \ldots, l_{n}\right\}$ of line momenta contains a basis of $\mathscr{L}$ with respect to $k$. Let $z=\left(z_{1}, \ldots, z_{n}\right) \in \mathbf{Z}^{4 n}$ and $k \in \mathbf{R}^{4 m}$ satisfying

$$
K_{i}(k)=\sum_{j=1}^{m} C_{i j} k_{j}=\frac{2 \pi}{a} z_{i}, \quad i=1, \ldots, n .
$$

By $\operatorname{rank}\left(C_{i j}\right)=m$, this system has a unique solution. By naturalness of $\mathscr{L}$, it is of the form $k=\frac{2 \pi}{a} \Delta, \Delta \in \mathbf{Z}^{4 m}$ (Appendix D). For $k \in[-\pi / a, \pi / a]^{4 m}$ this is possible only if $\Delta=0$, i.e., $z=0$. Hence, having chosen $\varepsilon>0$ according to Lemma D.1, for $J_{0}$ only $z$ $=0$ appears in (4.3). The integrand of $\bar{I}_{J_{0} 0}^{0}$ and hence of $\hat{I}_{J_{0} 0}^{0}$ is bounded by

and the integral

$$
\alpha^{n} \frac{|P(k, q)|}{\prod_{i=1}^{n}\left(l_{i}^{2}(k, q)+\mu_{i}^{2}\right)},
$$

$$
\alpha^{n} \int_{-\infty}^{\infty} d^{4} k_{1} \ldots d^{4} k_{m} \frac{|P(k, q)|}{\prod_{i=1}^{n}\left(l_{i}^{2}(k, q)+\mu_{i}^{2}\right)}
$$

is convergent by (7.15) and the power counting theorem of [1] (or by the auxiliary power counting theorem). Using Lebesgue's "theorem of dominated convergence", 
we get

$$
\lim _{a \rightarrow 0} \hat{I}(q ; \mu, a)=\lim _{a \rightarrow 0} \hat{I}_{J_{0} 0}^{0}(q ; \mu, a)=\int_{-\infty}^{\infty} d^{4} k_{1} \ldots d^{4} k_{m} \frac{P(k, q)}{\prod_{i=1}^{n}\left(l_{i}^{2}(k, q)+\mu_{i}^{2}\right)} .
$$

This completely proves the power counting theorem.

\section{Some Technical Lemmas}

We now start to prove the auxiliary power counting theorem. The proof idea is similar to that of the convergence theorem of Hahn and Zimmermann [1]. However, to discuss the cutoff behavior, we need some deeper statements (e.g. Lemma 8.6). In this and the next section we shall use the notations of Sect. 5. Especially, $\mathscr{L}$ is a set as given in (5.1). In addition, throughout the sequel we shall use the shorthand notation

$$
J_{\lambda}\left(\left\{P_{i}\right\} \mid q, \mu\right)=\int^{\lambda, \mathscr{L}} d^{4} k_{1} \ldots d^{4} k_{m} \frac{\min _{i \in I} \lambda^{-p_{i}}\left|P_{i}(k)\right|}{E(k, q, \mu)} .
$$

Here, $I$ is a finite set, and $\left\{P_{i}\right\}$ represents a set of polynomials $P_{i}, i \in I$. For $\tau>0$ we define

$$
D^{q, \tau}=\left\{\left(k_{1}, \ldots, k_{m}\right) \in \mathbf{R}^{4 m} \mid l_{j}^{2}(k, q) \geqq \tau^{2} \quad \text { for all } \quad l_{j} \in \mathscr{L}\right\},
$$

and for $X \subseteq \mathbf{R}^{4 m}$

$$
\begin{aligned}
J_{\lambda}\left(\left\{P_{i}\right\} \mid q, \mu, X\right) & =\int_{X}^{\lambda, \mathscr{L}} d^{4} k_{1} \ldots d^{4} k_{m} \frac{\min _{i \in I} \lambda^{-p_{i}}\left|P_{i}(k)\right|}{E(k, q, \mu)} \\
& \equiv \int^{\lambda, \mathscr{L}} d^{4} k_{1} \ldots d^{4} k_{m} \chi_{X}(k) \frac{\min _{i \in I} \lambda^{-p_{i}}\left|P_{i}(k)\right|}{E(k, q, \mu)},
\end{aligned}
$$

where $\chi_{X}$ is the characteristic function

$$
\chi_{X}(k)= \begin{cases}1, & k \in X \\ 0, & k \notin X .\end{cases}
$$

The present section contains a series of lemmas which will be used to prove the auxiliary power counting theorem by induction on the number of (fourdimensional) integrations.

Lemma 8.1. Let I be a finite set and $r \in \mathbf{N}_{0}=\{0,1,2, \ldots\}$. For every $i \in I$ let $P_{i}(x, z)$ be a function of $x \in \mathbf{R}^{p}$ and a polynomial in variables $z_{j}, j=1, \ldots, n$ of degree smaller or equal to $r$ :

$$
P_{i}(x, z)=\sum_{j_{1}=0}^{r} \ldots \sum_{j_{n}=0}^{r} a_{j_{1} \ldots j_{n}}^{(i)}(x) z_{1}^{j_{1}} \ldots z_{n}^{j_{n}}
$$


Let $y_{0}, \ldots, y_{r} \in \mathbf{R}$ be $r+1$ different points, $Y=\left\{\left(z_{1}, \ldots, z_{n}\right) \mid z_{i} \in\left\{y_{0}, \ldots, y_{r}\right\}\right.$ for all $i$ $=1, \ldots, n\}$ and $\Omega \subseteq \mathbf{R}^{p}$. If the integrals

$$
\int_{\Omega} d x \min _{i \in I}\left|P_{i}\left(x, y^{(i)}\right)\right|
$$

are convergent for all $y^{(i)} \in Y$, then so are the integrals

$$
\int_{\Omega} d x \min _{i \in I}\left|a_{j_{i 1} \ldots j_{i n}}^{(i)}(x)\right|
$$

for arbitrary $j_{i 1}, \ldots, j_{i n} \in\{0, \ldots, r\}$, and there exists $c>0$, depending only on $y_{0}, \ldots, y_{r}$, such that

$$
\int_{\Omega} d x \min _{i \in I}\left|a_{j_{i 1} \ldots j_{i n}}^{(i)}(x)\right| \leqq c \cdot \sum_{y^{(i)} \in Y \text { for all }} \int_{i \in I} d x \min _{i \in I}\left|P_{i}\left(x, y^{(i)}\right)\right| .
$$

The number $c$ is independent of the integration domain $\Omega$.

Proof. For every $i \in I$ there exist constants $c_{j_{1} \ldots j_{n}}^{(i)}(y)$ such that

$$
a_{j_{1} \ldots j_{n}}^{(i)}(x)=\sum_{y \in Y} c_{j_{1} \ldots j_{n}}^{(i)}(y) \cdot P_{i}(x, y) .
$$

Using the inequality of Appendix B, (8.5b) follows, where

$$
c=\max _{j_{1}, \ldots, j_{n}, y \in Y, i \in I}\left|c_{j_{1} \ldots j_{n}}^{(i)}(y)\right| .
$$

The following lemma is a direct consequence of Lemma 8.1.

Lemma 8.2. Let I be a finite set and $r \in \mathbf{N}_{0}=\{0,1,2, \ldots\}$. Every polynomial $P_{i}$ of degree smaller or equal to $r$ in the components of $k_{1}, \ldots, k_{m}$ can be written as

$$
P_{i}(k)=\sum_{\alpha=0}^{r} P_{i \alpha}(k), \quad i \in I,
$$

where $P_{i \alpha}$ is a homogeneous polynomial of order $\alpha$. Let $\gamma_{0}, \ldots, \gamma_{r} \in \mathbf{R}$ be $r+1$ different points and $Y=\left\{\gamma_{0}, \ldots, \gamma_{r}\right\}$. Then there exists $c>0$, depending only on $\gamma_{0}, \ldots, \gamma_{r}$ (but not on the polynomials $P_{i}$ ), such that

$$
\begin{aligned}
& \int d^{4} k_{1} \ldots d^{4} k_{m} \frac{\min _{i \in I} \lambda^{-p_{i}}\left|P_{i \alpha_{i}}(k)\right|}{E(k, q, \mu)} \\
& \leqq c \sum_{\varrho_{\imath} \in Y \text { for all }} \int_{i \in I}^{\lambda, \mathscr{L}} d^{4} k_{1} \ldots d^{4} k_{m} \frac{\min _{i \in I} \lambda^{-p_{i}}\left|P_{i}\left(\varrho_{i} k\right)\right|}{E(k, q, \mu)},
\end{aligned}
$$

for arbitrary sequences $\left(\mathrm{P}_{i \alpha_{\imath}}\right)_{i \in I}$.

Proof. Since $\operatorname{rank}_{k} \mathscr{L}=m$, the integral

$$
\int d^{4} k_{1} \ldots d^{4} k_{m} \frac{\min _{i \in I} \lambda^{-p_{i}}\left|\sum_{\alpha=0}^{r} \varrho_{i}^{\alpha} P_{i \alpha}(k)\right|}{E(k, q, \mu)}
$$


is convergent for every finite $\lambda$ and all $\varrho_{i} \in \mathbf{R}, i \in I$. Since $\gamma_{0}, \ldots, \gamma_{r} \in \mathbf{R}$ are different points and $Y=\left\{\gamma_{0}, \ldots, \gamma_{r}\right\}$, Lemma 8.1 implies that

$$
\begin{aligned}
& \int d^{4} k_{1} \ldots d^{4} k_{m} \frac{\min _{i \in I} \lambda^{-p_{\imath}}\left|P_{i \alpha_{i}}(k)\right|}{E(k, q, \mu)} \\
& \leqq c \sum_{\varrho_{\imath} \in Y \text { for all }} \int_{i \in I}^{\lambda, \mathscr{L}} d^{4} k_{1} \ldots d^{4} k_{m} \frac{\min _{i \in I} \lambda^{-p_{i}}\left|\sum_{\alpha=0}^{r}\left(\varrho_{i}\right)^{\alpha} P_{i \alpha}(k)\right|}{E(k, q, \mu)} \\
& =c \sum_{\varrho_{i} \in Y \text { for all }}^{\lambda, \mathscr{L}} \int_{i \in I} d^{4} k_{1} \ldots d^{4} k_{m} \frac{\min _{i \in I} \lambda^{-p_{i}}\left|P_{i}\left(\varrho_{i} k\right)\right|}{E(k, q, \mu)}
\end{aligned}
$$

for some constant $c$ depending on $\gamma_{0}, \ldots, \gamma_{r}$ only.

Next we quote

Lemma 8.3 [1]. Let $k, l \in \mathbf{R}^{4}$ and $\mu>0$. Then

$$
\frac{(k+l)^{2}+\mu^{2}}{k^{2}+\mu^{2}} \leqq c(l) \text { and } \frac{k^{2}+\mu^{2}}{(k+l)^{2}+\mu^{2}} \leqq c(l) \text {, }
$$

where $c(l)=1+\|l\| / \mu+l^{2} / \mu^{2}$. where $c(l)=1+\|l\| \mu+l^{2} / \mu^{2}$.
Recall that we are using the Euclidean norm $\|l\|=\sqrt{\sum_{i=1}^{4} l_{i}^{2}}$ for $l \in \mathbf{R}^{4}$. As a
corollary, we have

Lemma 8.4. If the momenta $q$ are bounded, then there exists $c(\mu)>0$ such that

$$
\int^{\lambda, \mathscr{L}} d^{4} k_{1} \ldots d^{4} k_{m} \frac{\min _{i \in I} \lambda^{-p_{i}}\left|P_{i}(k)\right|}{E(k, q, \mu)} \leqq c(\mu) \int^{\lambda, \mathscr{L}} d^{4} k_{1} \ldots d^{4} k_{m} \frac{\min _{i \in I} \lambda^{-p_{i}}\left|P_{i}(k)\right|}{E(k, 0, \mu)} .
$$

Excluding small neighborhoods of the poles of the propagators, i.e., regions where some line momenta vanish, the masses may be set to zero without affecting the large cutoff behavior:

Lemma 8.5. For $\tau>0$

1.

$$
J_{\lambda}\left(\left\{P_{i}\right\} \mid q, \mu, D^{q, \tau}\right) \leqq J_{\lambda}\left(\left\{P_{i}\right\} \mid q, 0, D^{q, \tau}\right) .
$$

2. There exists a $c(\mu, \tau)>0$ such that

$$
J_{\lambda}\left(\left\{P_{i}\right\} \mid q, 0, D^{q, \tau}\right) \leqq c(\mu, \tau) J_{\lambda}\left(\left\{P_{i}\right\} \mid q, \mu, D^{q, \tau}\right) .
$$

This can be seen from $l^{2} /\left(l^{2}+\mu^{2}\right) \leqq 1$ and $\left(l^{2}+\mu^{2}\right) / l^{2} \leqq 1+\left(\mu^{2} / \tau^{2}\right)$. We will now show that the cutoff dependence of $J_{\lambda}$ does not change if the poles of propagators are excluded from the integration domain (Lemma 8.8). This lemma will be used in Lemma 8.9 to get homogeneous denominators in $J_{\lambda}$. As a preliminary, we state 
Lemma 8.6. Let $I$ be a finite set, $r \in \mathbf{N}_{0}=\{0,1,2, \ldots\}$ and $\tau>0$. Set

$$
\begin{aligned}
& X_{\lambda}^{q, \tau}=\left\{\left(k_{1}, \ldots, k_{m}\right) \in \mathbf{R}^{4 m} \mid \begin{array}{rl}
\left\|l_{j}(k, q)\right\| \leqq \tau ; & j=1, \ldots, s \\
\tau \leqq\left\|l_{j}(k, q)\right\| \leqq \lambda ; & j=s+1, \ldots, N
\end{array}\right\}, \\
& D_{\lambda}^{q, \tau}=\left\{\left(k_{1}, \ldots, k_{m}\right) \in \mathbf{R}^{4 m} \mid \tau \leqq\left\|l_{j}(k, q)\right\| \leqq \lambda ; \quad j=1, \ldots, N\right\} .
\end{aligned}
$$

Suppose $\left\{l_{1}, \ldots, l_{s}\right\}$ contains a basis of $\mathscr{L}$ with respect to $k$. Then there exist $\bar{a}(C, \tau)>0$ and $c_{0}(C, \mu, r, \tau)>0$ such that for all polynomials $P_{i}$ of degree $r_{i}$, constrained by $\sum_{i \in I} r_{i} \leqq r$, we have

$$
\begin{aligned}
& \int_{X_{\lambda}^{q, \tau}} d^{4} k_{1} \ldots d^{4} k_{m} \frac{\min _{i \in I} \lambda^{-p_{i}}\left|P_{i}(k)\right|}{\prod_{\mathscr{N}}\left(l_{j}^{2}(k, q)+\mu_{j}^{2}\right)^{n_{j}}} \\
& \quad \leqq c_{0}(C, \mu, r, \tau) \int_{D_{\bar{\lambda}, \tau}} d^{4} k_{1} \ldots d^{4} k_{m} \frac{\min _{i \in I} \lambda^{-p_{2}}\left|P_{i}(k)\right|}{\prod_{\mathscr{N}}\left(l_{j}^{2}(k, q)+\mu_{j}^{2}\right)^{n_{j}}},
\end{aligned}
$$

where $\bar{\lambda}=\lambda+\bar{a}(C, \tau)$.

Note that $c_{0}$ and $\bar{a}$ are independent of external momenta $q$.

Proof. The set $\mathscr{L}$ is given in (5.1). First of all assume

$$
C_{i j}=\delta_{i j}, \quad Q_{i}=0 \text { for every } i=1, \ldots, m .
$$

Then $X_{\lambda}^{q, \tau}$ is the set of all $\left(k_{1}, \ldots, k_{m}\right) \in \mathbf{R}^{4 m}$ satisfying $\left\|k_{i}\right\| \leqq \tau$ for $i=1, \ldots, m$ and

$$
\begin{array}{r}
\left\|\sum_{j=1}^{m} C_{i j} k_{j}+Q_{i}\right\| \leqq \tau, \quad i=m+1, \ldots, s, \\
\tau \leqq\left\|\sum_{j=1}^{m} C_{i j} k_{j}+Q_{i}\right\| \leqq \lambda, \quad i=s+1, \ldots, N,
\end{array}
$$

and $D_{\bar{\lambda}}^{q, \tau}$ is the set of $k$ satisfying $\tau \leqq\left\|k_{i}\right\| \leqq \bar{\lambda}$ for $i=1, \ldots, m$ and

$$
\tau \leqq\left\|\sum_{j=1}^{m} C_{i j} k_{j}+Q_{i}\right\| \leqq \bar{\lambda}, \quad i=m+1, \ldots, N .
$$

To prove Lemma 8.6 we use the following lemma proved in Appendix A.

Lemma A.1. Let I be a finite set, $r \in \mathbf{N}_{0}$ and $\sigma_{1}, \sigma_{2}$ compact cubes in $\mathbf{R}^{4 m}, \sigma_{2}$ containing an open set. Then there exists a constant $c\left(\sigma_{1}, \sigma_{2}, C, \mu, r\right)>0$ such that

$$
\begin{aligned}
& \int_{\sigma_{1}} d^{4} k_{1} \ldots d^{4} k_{m} \frac{\min _{i \in I}\left|P_{i}(k)\right|}{\prod_{\mathscr{N}}\left(l_{i}^{2}(k, q)+\mu_{i}^{2}\right)^{n_{1}}} \\
& \quad \leqq c\left(\sigma_{1}, \sigma_{2}, C, \mu, r\right) \int_{\sigma_{2}} d^{4} k_{1} \ldots d^{4} k_{m} \frac{\min _{i \in I}\left|P_{i}(k)\right|}{\prod_{\mathscr{N}}\left(l_{i}^{2}(k, q)+\mu_{i}^{2}\right)^{n_{j}}}
\end{aligned}
$$


for arbitrary polynomials $P_{i}$ of degree $r_{i}$, constrained by $\sum_{i \in I} r_{i} \leqq r$, and for all mo-
menta $q$.

To apply this lemma we have to find $\bar{a}>0$ and cubes $\sigma_{1}, \sigma_{2}$ having the desired properties and satisfying

$$
X_{\lambda}^{q, \tau} \subseteq \sigma_{1} \quad \text { and } \quad \sigma_{2} \subseteq D_{\lambda}^{q, \tau},
$$

where $\bar{\lambda}=\lambda+\bar{a}$. At first, Lemma 8.6 is trivial if $X_{\lambda}^{q, \tau}=\emptyset$. Furthermore, if $N=m, X_{\lambda}^{q, \tau}$ and $D_{\lambda}^{q, \tau}$ are independent of $q$, and $D_{\lambda}^{q, \tau}$ for $\lambda \geqq 2 \tau$ contains an open subset of $\mathbf{R}^{4 m}$ which is independent of $\lambda$. Hence Lemma A. 1 is applicable, and for $\bar{a}=2 \tau$, Lemma 8.6 follows.

Let $N \geqq m+1$ and $X_{\lambda}^{q, \tau} \neq \emptyset$. We now proceed to construct appropriate cubes $\sigma_{1}$, $\sigma_{2}$ in several steps.

i) $X_{\lambda}^{q, \tau} \subseteq \sigma_{1}$, where $\sigma_{1}=\prod_{i=1}^{m}[-\tau, \tau]^{4}$.

ii) There exists $R(C, \tau)>0$ such that $\left\|Q_{i}\right\| \leqq \lambda+R(C, \tau)$ for every $i=m$ $+1, \ldots, N$. For, setting $\bar{C}=\max _{i, j}\left|C_{i j}\right|, R=\bar{C} m \tau$, and $\left(k_{1}, \ldots, k_{m}\right) \in X_{\lambda}^{q, \tau}$, we obtain

$$
\left\|Q_{i}\right\|=\left\|\sum_{j=1}^{m} C_{i j} k_{j}+Q_{i}-\sum_{j=1}^{m} C_{i j} k_{j}\right\| \leqq \lambda+R(C, \tau) ; \quad i=m+1, \ldots, N .
$$

Next, we define

$$
\begin{aligned}
\Delta= & \left\{Q=\left(Q_{m+1}, \ldots, Q_{N}\right) \in \mathbf{R}^{4(N-m)} \mid\left\|Q_{i}\right\| \leqq \lambda\right. \\
& +R(C, \tau), \quad i=m+1, \ldots, N\} .
\end{aligned}
$$

If $\lambda$ grows, $Q$ is not bounded, hence a cube $\sigma_{2}$ contained in $D_{\bar{\lambda}}^{q}, \tau$ for all values of $Q$ does not exist. Instead, we construct $\bar{a}(C, \tau)>0$ and a finite set of cubes, so that for every $Q \in \Delta$ one of them is contained in $D_{\bar{\lambda}}^{q, \tau}$, where $\bar{\lambda}=\lambda+\bar{a}$.

iii) To this end, we construct numbers $b_{0}(C, \tau), \ldots, b_{N-m+1}(C, \tau)$ as follows. Set $b_{0}(C, \tau)=0$. If $b_{0}, \ldots, b_{r-1}$ are given for an integer $r, 1 \leqq r \leqq N-m+1$, choose $b_{r}(C, \tau)>0$ such that the set of $\left(k_{1}, \ldots, k_{m}\right) \in \mathbf{R}^{4 m}$, satisfying

$$
\begin{array}{cl}
\tau \leqq\left\|k_{i}\right\|, & i=1, \ldots, m, \\
b_{r-1}(C, m, \tau)+2 \tau \leqq\left\|\sum_{j=1}^{m} C_{i j} k_{j}\right\| \leqq b_{r}(C, \tau), & i=m+1, \ldots, N
\end{array}
$$

contains a compact cube $\Omega_{r}$ which itself contains an open set. Such numbers $b_{0}, \ldots, \dot{b}_{N-m+1}$ do always exist, $\sum_{j=1}^{m} C_{i j} k_{j}=0$ being a hyperplane in $\mathbf{R}^{4 m}$.

iv) Consider the following subsets of $\Delta$ :

a) $\Delta_{N-m+1} \subseteq \Delta$ such that $\left\|Q_{i}\right\|<b_{N-m}+\tau$ for all $i=m+1, \ldots, N$.

b) For $r, 1 \leqq r \leqq N-m$, let $\Delta_{r} \subseteq \Delta$ such that for every $i=m+1, \ldots, N$

$$
\left\|Q_{i}\right\|<b_{r-1}+\tau \text { or }\left\|Q_{i}\right\| \geqq b_{r}+\tau \text {. }
$$

Obviously, $\Delta$ is the union of these sets. For every $r$ let $K_{r}(C, \tau)$ be a number such that $\left\|k_{i}\right\| \leqq K_{r}(C, \tau)$ for all $\left(k_{1}, \ldots, k_{m}\right) \in \Omega_{r}$. Set $a_{r}(C, \tau)=\max \left(K_{r}(C, \tau), b_{r}(C, \tau)\right.$ $+R(C, \tau))$ and $\bar{\lambda}=\lambda+a_{r}$. Then for $Q \in \Delta_{r}$, using (8.24), we easily get $\Omega_{r} \subseteq D_{\bar{\lambda}}^{q, \tau}$. 
v) Let $\tilde{\Omega}$ be the finite set of cubes constructed in iv), and $\bar{a}(C, \tau)=\max a_{r}$. We have just shown that for every $Q \in \Delta$ there exists a cube $\sigma \in \widetilde{\Omega}$ which is contained in $D_{\bar{\lambda}}^{q, \tau}$. By Lemma A.1, for every $\sigma$ there is a constant $c\left(\sigma_{1}, \sigma, C, r, \tau\right)>0$ such that

$$
\begin{aligned}
& \int_{X_{\lambda}^{q, \tau}} d^{4} k_{1} \ldots d^{4} k_{m} \frac{\min _{i \in I} \lambda^{-p_{\imath}}\left|P_{i}(k)\right|}{\prod_{\mathscr{N}}\left(l_{j}^{2}(k, q)+\mu_{j}^{2}\right)^{n_{j}}} \\
& \leqq \int_{\sigma_{1}} d^{4} k_{1} \ldots d^{4} k_{m} \frac{\min _{i \in I} \lambda^{-p_{i}}\left|P_{i}(k)\right|}{\prod_{\mathscr{N}}\left(l_{j}^{2}(k, q)+\mu_{j}^{2}\right)^{n_{j}}} \\
& \leqq c\left(\sigma_{1}, \sigma, C, \mu, r\right) \int_{\sigma} d^{4} k_{1} \ldots d^{4} k_{m} \frac{\min _{i \in I} \lambda^{-p_{\imath}}\left|P_{i}(k)\right|}{\prod_{\mathscr{N}}\left(l_{i}^{2}(k, q)+\mu_{i}^{2}\right)^{n_{J}}} \\
& \left.\left.\leqq c_{0}(C, \mu, r, \tau) \int_{D_{\lambda}^{q_{\lambda} \tau}} d^{4} k_{1} \ldots d^{4} k_{m} \frac{\min _{i \in I} \lambda^{-p_{i}}\left|P_{i}(k)\right|}{\prod_{\mathscr{N}}\left(l_{i}^{2}(k, q)+\mu_{i}^{2}\right)^{n_{J}}} \quad \text { (by iv }\right)\right),
\end{aligned}
$$

where $c_{0}(C, \mu, r, \tau)=\max c\left(\sigma_{1}, \sigma, C, \mu, r\right)$ and $\bar{\lambda}=\lambda+\bar{a}$. This proves Lemma 8.6, if (8.16) holds. $\sigma \in \widetilde{\Omega}$

In the general case, we make a non-singular transformation

$$
\begin{gathered}
k_{1}^{\prime}=l_{i_{1}}(k, q)=\sum_{j=1}^{m} C_{i_{1} j} k_{j}+Q_{i_{1}}(q) \\
\vdots \\
k_{m}^{\prime}=l_{i_{m}}(k, q)=\sum_{j=1}^{m} C_{i_{m j}} k_{j}+Q_{i_{m}}(q) .
\end{gathered}
$$

This is always possible because $\left\{l_{1}, \ldots, l_{s}\right\}$ contains a basis $\left\{l_{i_{1}}, \ldots, l_{i_{m}}\right\}$ of $\mathscr{L}$ (with respect to $k$ ). Under such a transformation, the form of (8.15) does not change. Every $l_{i} \in \mathscr{L}$ has the form

$$
l_{i}\left(k^{\prime}, q\right)=\sum_{j=1}^{m} \bar{C}_{i j} k_{j}^{\prime}+\bar{Q}_{i}(q), \quad i=1, \ldots, N,
$$

and $\bar{C}$ satisfies (5.1b) and (8.16). This reduces the general case to the above situation, and the lemma is proved.

We now generalize Lemma 8.6 to arbitrary "sectors" $X_{\mathscr{S}}^{\tau}$. For any $\mathscr{S} \subseteq \mathscr{L}, X_{\mathscr{S}}^{\tau}$ denotes the set of $k \in \mathbf{R}^{4 m}$ satisfying

$$
\begin{array}{ll}
l_{j}^{2}(k, q) \geqq \tau^{2} \quad \text { for } \quad l_{j} \in \mathscr{P}, \\
l_{j}^{2}(k, q) \leqq \tau^{2} \quad \text { for } \quad l_{j} \in \mathscr{L} \backslash \mathscr{S} .
\end{array}
$$

Lemma 8.7. Let $I$ be a finite set, $r \in \mathbf{N}_{0}=\{0,1,2, \ldots\}$ and $\tau>0$. Then there exist $K(\tau)>0$ and $c(\mu, r, \tau)>0$, so that for arbitrary polynomials $P_{i}$ of degree $r_{i}$ in the 

components of $k_{1}, \ldots, k_{m}$, constrained by $\sum_{i \in I} r_{i} \leqq r$, in every sector $X_{\mathscr{S}}^{\tau}$ and for all
$\lambda>K(\tau)$, the bound

$$
J_{\lambda}\left(\left\{P_{i}\right\} \mid q, \mu, X_{\mathscr{S}}^{\tau}\right) \leqq c(\mu, r, \tau) J_{\bar{\lambda}}\left(\left\{P_{i}\right\} \mid q, \mu, D^{q, \tau}\right)
$$

holds, where $\bar{\lambda}=\lambda+K(\tau)$.

The set $D^{q, \tau}$ is defined in (8.2). The constants $K$ and $c$ are independent of external momenta $q$. In general we suppress the dependence on the incidence matrix $C$. On the contrary, mass dependence will be written explicitly, since non-vanishing masses are important to avoid IR-singularities.

Proof. Let $\mathscr{P} \subseteq \mathscr{L}$ be an arbitrary subset and $X_{\mathscr{S}}^{\tau}$ the corresponding sector. If $\mathscr{S}=\mathscr{L}$, the statement is trivial. Hence let $\mathscr{S} \neq \mathscr{L}$. By an appropriate renumbering, $X_{\mathscr{S}}^{\tau}$ is the set of $k \in \mathbf{R}^{4 m}$ satisfying

$$
\begin{aligned}
& l_{j}^{2}(k, q) \geqq \tau^{2} \quad \text { for } \quad j=1, \ldots, \alpha, \\
& l_{j}^{2}(k, q) \leqq \tau^{2} \quad \text { for } \quad j=\alpha+1, \ldots, N,
\end{aligned}
$$

where $\alpha \in \mathbf{N}_{0}$. Let us write $l_{j}(k, q)=K_{j}(k)+Q_{j}(q)$ for every $j=1, \ldots, N$. Renumbering again, one can find $b \geqq \alpha+1$ and $a, \gamma, 1 \leqq a \leqq \gamma \leqq \alpha$, so that the following conditions hold.

1. $\overline{\bar{K}}=\left\{K_{\alpha+1}, \ldots, K_{b}\right\}$ is a basis of $\left\{K_{\alpha+1}, \ldots, K_{N}\right\}$.

2. It can be completed by $\bar{K}=\left\{K_{1}, \ldots, K_{a}\right\}$ to a basis of $\left\{K_{1}, \ldots, K_{N}\right\}$.

3. For every $\beta=1, \ldots, \gamma$,

and $\gamma$ is maximal.

$$
K_{\beta}=K_{\beta}(\bar{K})=\sum_{i=1}^{a} c_{\beta i} K_{i}, \quad c_{\beta i} \in \mathbf{R}
$$

Then, for $\beta=\gamma+1, \ldots, N$,

$$
K_{\beta}=K_{\beta}(\bar{K}, \bar{K})=\sum_{i=\alpha+1}^{b} d_{\beta i} K_{i}+\sum_{i=1}^{a} f_{\beta i} K_{i} ; \quad\left(d_{\beta(\alpha+1)}, \ldots, d_{\beta b}\right) \neq 0, \quad d_{\beta i}, f_{\beta i} \in \mathbb{R} .
$$

Define

$$
Z_{\mathscr{S}}^{\tau, \lambda}=\left\{\bar{K}=\left(K_{1}, \ldots, K_{a}\right) \in \mathbf{R}^{4 a} \mid r^{2} \leqq\left(K_{j}(\bar{K})+Q_{j}\right)^{2} \leqq \lambda^{2} ; j=1, \ldots, \gamma\right\},
$$

and for any $\bar{K}$ let $X_{\mathscr{S} \bar{K}}^{\tau, \lambda}$ be the set of all $\bar{K}=\left(K_{\alpha+1}, \ldots, K_{b}\right) \in \mathbf{R}^{4(b-x)}$ such that

$$
\begin{array}{cc}
\left(K_{j}(\bar{K})+Q_{j}\right)^{2} \leqq \tau^{2} ; & j=\alpha+1, \ldots, N, \\
\tau^{2} \leqq\left(K_{j}(\bar{K}, \bar{K})+Q_{j}\right)^{2} \leqq \lambda^{2} ; & j=\gamma+1, \ldots, \alpha .
\end{array}
$$

Using $\bar{K}, \overline{\bar{K}}$ as new integration variables in $J_{\lambda}\left(\left\{P_{i}\right\} \mid q, \mu, X_{\mathscr{S}}^{\tau}\right)$, we get

$$
J_{\lambda}\left(\left\{P_{i}\right\} \mid q, \mu, X_{\mathscr{S}}^{\tau}\right)=d_{\mathscr{S}} \int_{Z_{\mathscr{S}}^{\tau, \lambda}} d \bar{K} \frac{1}{E_{1}(k(\bar{K}), q, \mu)} \int_{X_{\mathscr{S}, \bar{K}}^{\tau, \lambda}} d \overline{\bar{K}} \frac{\min _{i \in I} \lambda^{-p_{i}}\left|P_{i}(k(\bar{K}, \bar{K}))\right|}{E_{2}(k(\bar{K}, \overline{\bar{K}}), q, \mu)},
$$

where

$$
\begin{array}{ll}
E_{1}(k(\bar{K}), q, \mu)=\prod_{\mathscr{N}_{1}}\left(l_{j}^{2}(k(\bar{K}), q)+\mu_{j}^{2}\right)^{n_{j}} ; & \mathscr{N}_{1}=\mathscr{N} \cap\left\{l_{j} \in \mathscr{L} \mid j=1, \ldots, \gamma\right\}, \\
E_{2}(k(\bar{K}, \bar{K}), q, \mu)=\prod_{\mathscr{N}_{2}}\left(l_{j}^{2}(k(\bar{K}, \bar{K}), q)+\mu_{j}^{2}\right)^{n_{J}} ; & \mathscr{N}_{2}=\mathscr{N} \cap\left\{l_{j} \in \mathscr{L} \mid j=\gamma+1, \ldots, N\right\} .
\end{array}
$$


$d_{\mathscr{S}}$ is the Jacobian of $\left(k_{1}, \ldots, k_{m}\right)$ with respect to $(\bar{K}, \bar{K})$. We can now apply Lemma 8.6 to the inner integral in (8.30). For, the set of momenta

$$
l_{j}(k(\bar{K}, \overline{\bar{K}}), q)=K_{j}(\bar{K}, \overline{\bar{K}})+Q_{j}(q), \quad j=\alpha+1, \ldots, N
$$

contains a basis of $\left\{l_{\gamma+1}, \ldots, l_{N}\right\}$ with respect to $\bar{K}$. Hence there exist $K_{\mathscr{S}}(\tau)>0$ and $c_{\mathscr{S}}(\mu, r, \tau)>0$, so that

$$
\int_{X_{\overline{\mathscr{S}}, \overline{\bar{K}}}^{\tau}} d \overline{\bar{K}} \frac{\min _{i \in I} \lambda^{-p_{i}}\left|P_{i}(k(\bar{K}, \overline{\bar{K}}))\right|}{E_{2}(k(\bar{K}, \overline{\bar{K}}), q, \mu)} \leqq c_{\mathscr{S}}(\mu, r, \tau) \int_{D_{D_{\mathscr{Y}}^{\tau, \overline{\bar{K}}}}} d \overline{\bar{K}} \frac{\min _{i \in I} \lambda^{-p_{i}}\left|P_{i}(k(\bar{K}, \overline{\bar{K}}))\right|}{E_{2}(k(\bar{K}, \overline{\bar{K}}), q, \mu)}
$$

for all polynomials $P_{i}$ of degree $r_{i}, \sum_{i \in I} r_{i} \leqq r$, where $\bar{\lambda}=\lambda+K_{\mathscr{S}}(\tau)$ and

$$
\begin{aligned}
D_{\mathscr{S} \bar{K}}^{\tau, \bar{\lambda}} & =\left\{\overline{\bar{K}}=\left(K_{\alpha+1}, \ldots, K_{b}\right) \in \mathbf{R}^{4(b-\alpha)} \mid \tau^{2}\right. \\
& \left.\leqq\left(K_{j}(\bar{K}, \overline{\bar{K}})+Q_{j}\right)^{2} \leqq \bar{\lambda}^{2} ; \quad j=\gamma+1, \ldots, N\right\} .
\end{aligned}
$$

Consequently

$$
\begin{aligned}
& J_{\lambda}\left(\left\{P_{i}\right\} \mid q, \mu, X_{\mathscr{S}}^{\tau}\right) \leqq c_{\mathscr{S}}(\mu, r, \tau) d_{\mathscr{S}} \int_{Z_{\mathscr{Y}}^{\tau, \overline{ }}} d \bar{K} \int_{D_{\bar{S}_{\bar{K}} \overline{\bar{K}}}} d \overline{\bar{K}} \frac{\min _{i \in I} \lambda^{-p_{i}}\left|P_{i}(k(\bar{K}, \overline{\bar{K}}))\right|}{E(k(\bar{K}, \overline{\bar{K}}), q, \mu)} \\
& =c_{\mathscr{S}}(\mu, r, \tau) \int_{D^{q, \tau}}^{\bar{\lambda}, \mathscr{L}} d^{4} k_{1} \ldots d^{4} k_{m} \frac{\min _{i \in I} \lambda^{-p_{\imath}}\left|P_{i}(k)\right|}{E(k, q, \mu)} .
\end{aligned}
$$

Setting $K(\tau)=\max _{\mathscr{S} \subset \mathscr{L}} K_{\mathscr{S}}(\tau)$ and using

$$
\lambda^{-p_{\imath}} \leqq(\bar{\lambda})^{-p_{\imath}} \cdot 2^{p_{i}},
$$

for $\lambda>K(\tau)$ and $\bar{\lambda}=\lambda+K(\tau)$, one can find $c(\mu, r, \tau)>0$, so that

$$
\begin{aligned}
J_{\lambda}\left(\left\{P_{i}\right\} \mid q, \mu, X_{\mathscr{S}}^{\tau}\right) & \leqq c(\mu, r, \tau) \int_{D^{q, \tau}}^{\bar{\lambda}, \mathscr{L}} d^{4} k_{1} \ldots d^{4} k_{m} \frac{\min _{i \in I}(\bar{\lambda})^{-p_{\imath}}\left|P_{i}(k)\right|}{E(k, q, \mu)} \\
& =c(\mu, r, \tau) J_{\bar{\lambda}}\left(\left\{P_{i}\right\} \mid q, \mu, D^{q, \tau}\right) . \square
\end{aligned}
$$

As a corollary, we get

Lemma 8.8. Let $I$ be a finite set, $r \in \mathbf{N}_{0}=\{0,1,2, \ldots\}$ and $\tau>0$. Then

$$
J_{\lambda}\left(\left\{P_{i}\right\} \mid q, \mu, D^{q, \tau}\right) \leqq J_{\lambda}\left(\left\{P_{i}\right\} \mid q, \mu\right) .
$$

2. There exist $K(\tau)>0$ and $c(\mu, r, \tau)>0$, so that for all $\lambda>K(\tau)$ and all polynomials $P_{i}, i \in I$, of a degree $r_{i}$ in the components of $k_{1}, \ldots, k_{m}$, constrained by $\sum_{i \in I} r_{i} \leqq r$, we have

$$
J_{\lambda}\left(\left\{P_{i}\right\} \mid q, \mu\right) \leqq c(\mu, r, \tau) J_{\bar{\lambda}}\left(\left\{P_{i}\right\} \mid q, \mu, D^{q, \tau}\right),
$$

where $\bar{\lambda}=\lambda+K(\tau)$. 
Proof. The first statement is trivial. To prove 2, wirte $\mathbf{R}^{4 m}=\cup_{\mathscr{S}} X_{\mathscr{S}}^{\tau}$. If $\mathscr{S} \neq \mathscr{S}^{\prime}$, $X_{\mathscr{S}}^{\tau} \cap X_{\mathscr{S}}^{\tau}$, is a set of measure zero, hence

$$
J_{\lambda}\left(\left\{P_{i}\right\} \mid q, \mu\right)=\sum_{\mathscr{S}} J_{\lambda}\left(\left\{P_{i}\right\} \mid q, \mu, X_{\mathscr{S}}^{\tau}\right) .
$$

Using Lemma 8.7, there exist $K(\tau)>0$ and $c_{0}(\mu, r, \tau)>0$, so that for $\lambda>K(\tau)$ and every $\mathscr{S} \subseteq \mathscr{L}$,

$$
J_{\lambda}\left(\left\{P_{i}\right\} \mid q, \mu, X_{\mathscr{S}}^{\tau}\right) \leqq c_{0}(\mu, r, \tau) J_{\bar{\lambda}}\left(\left\{P_{i}\right\} \mid q, \mu, D^{q, \tau}\right),
$$

where $\bar{\lambda}=\lambda+K(\tau)$. The right-hand side is independent of $\mathscr{S}$. Summation over $\mathscr{S}$ then proves the assertion.

Using Lemma 8.8, we state the important

Lemma 8.9. Let $I$ be a finite set, $r \in \mathbf{N}_{0}=\{0,1,2, \ldots\}$ and $\tau>0$. Then

1. There exists $c_{1}(\mu, \tau)>0$ such that

$$
J_{\lambda}\left(\left\{P_{i}\right\} \mid q=0, \mu=0, D^{0, \tau}\right) \leqq c_{1}(\mu, \tau) J_{\lambda}\left(\left\{P_{i}\right\} \mid q=0, \mu\right) .
$$

2. One can find $K(\tau)>0$ and $c_{2}(\mu, r, \tau)>0$, so that for all polynomials $P_{i}$ of degree $r_{i}$, constrained by $\sum_{i \in I} r_{i} \leqq r$, and for all $\lambda>K(\tau)$, we have

$$
J_{\lambda}\left(\left\{P_{i}\right\} \mid q=0, \mu\right) \leqq c_{2}(\mu, r, \tau) J_{\bar{\lambda}}\left(\left\{P_{i}\right\} \mid q=0, \mu=0, D^{0, \tau}\right),
$$

where $\bar{\lambda}=\lambda+K(\tau)$.

3. Let $q$ be bounded. Then there exist $R>0$ and $c_{3}(\mu)>0$, so that for all $\lambda>R$

$$
J_{\lambda}\left(\left\{P_{i}\right\} \mid q, \mu\right) \leqq c_{3}(\mu) J_{\lambda}\left(\left\{P_{i}\right\} \mid q=0, \mu\right)
$$

where $\tilde{\lambda}=\lambda+R$.

Note $D^{0, \tau}=\left.D^{q, \tau}\right|_{q=0}$, and $D^{q, \tau}$ is defined in (8.2). If all masses are positive, external momenta do not have any influence on the cutoff dependence of $J_{\lambda}$. Proof. 1.

$$
\begin{aligned}
J_{\lambda}\left(\left\{P_{i}\right\} \mid q=0, \mu=0, D^{0, \tau}\right) & \leqq c_{1}(\mu, \tau) J_{\lambda}\left(\left\{P_{i}\right\} \mid q=0, \mu, D^{0, \tau}\right) & & (\text { by Lemma 8.5) } \\
& \leqq c_{1}(\mu, \tau) J_{\lambda}\left(\left\{P_{i}\right\} \mid q=0, \mu\right) & & \text { (by Lemma 8.8) } .
\end{aligned}
$$

2. Using Lemma 8.8, there exist $K(\tau)>0$ and $c_{2}(\mu, r, \tau)>0$, so that for all $\lambda>K(\tau)$,

$$
\begin{aligned}
J_{\lambda}\left(\left\{P_{i}\right\} \mid q=0, \mu\right) & \leqq c_{2}(\mu, r, \tau) J_{\bar{\lambda}}\left(\left\{P_{i}\right\} \mid q=0, \mu, D^{0, \tau}\right) \\
& \leqq c_{2}(\mu, r, \tau) J_{\bar{\lambda}}\left(\left\{P_{i}\right\} \mid q=0, \mu=0, D^{0, \tau}\right),
\end{aligned}
$$

where $\bar{\lambda}=\lambda+K(\tau)$, and we have used Lemma 8.5 again.

3. For $q$ in a bounded region we get by Lemma 8.4

$$
J_{\lambda}\left(\left\{P_{i}\right\} \mid q, \mu\right) \leqq c^{\prime}(\mu) \cdot \int^{\lambda, \mathscr{L}} d^{4} k_{1} \ldots d^{4} k_{m} \frac{\min _{i \in I} \lambda^{-p_{i}}\left|P_{i}(k)\right|}{E(k, 0, \mu)} .
$$


$\mathscr{L}$ is given in (5.1). Choose $R \geqq \max _{i=1, \ldots, N}\left\|Q_{i}(q)\right\|$ independent of $q$ and $\tilde{\lambda}=\lambda+R$. Then for every $j=1, \ldots, N$ and all $k$ in the integration domain we have

$$
\left\|l_{j}(k, 0)\right\| \leqq\left\|l_{j}(k, q)\right\|+\left\|Q_{i}(q)\right\| \leqq \tilde{\lambda}
$$

Furthermore, for $\lambda>R$ the estimate

$$
\left.\min _{i \in I} \lambda^{-p_{i}}\left|P_{i}(k)\right| \leqq d \cdot \min _{i \in I}(\tilde{\lambda})^{-p_{i}} \mid P_{i}(k)\right\}
$$

holds, where $d$ is a constant. Hence, setting $\overline{\mathscr{L}}=\left\{l_{i}(k, 0) \mid i=1, \ldots, N\right\}$ and $c_{3}(\mu)=c^{\prime}(\mu) d$, we get

$$
\begin{aligned}
J_{\lambda}\left(\left\{P_{i}\right\} \mid q, \mu\right) & \leqq c_{3}(\mu) \cdot \int^{\tilde{\lambda}, \overline{\mathscr{L}}} d^{4} k_{1} \ldots d^{4} k_{m} \frac{\min _{i \in I}(\tilde{\lambda})^{-p_{i}}\left|P_{i}(k)\right|}{E(k, 0, \mu)} \\
& =c_{3}(\mu) J_{\tilde{\lambda}}\left(\left\{P_{i}\right\} \mid q=0, \mu\right) . \square
\end{aligned}
$$

Finally, we state the following elementary

Lemma 8.10. Let $P$ be a polynomial in variables $(u)=\left(u_{1}, \ldots, u_{a}\right),(v)=\left(v_{1}, \ldots, v_{b}\right)$ and $q$, and let $W(v, q)=\left(W_{1}(v, q), \ldots, W_{a}(v, q)\right), R(v, q)=\left(R_{1}(v, q), \ldots, R_{b}(v, q)\right)$ be linear functions, and $\varrho>0$. Then

$$
\overline{\operatorname{degr}}_{u \mid v} P(\varrho u+W(v, q), R(v, q), q) \leqq \overline{\operatorname{degr}}_{u \mid v} P(u, v, q) .
$$

Proof. Write

$$
P(\varrho u+W(v, q), R(v, q), q)=\sum_{\alpha} S_{\alpha}(R(v, q), q) \cdot T_{\alpha}(\varrho u+W(v, q)),
$$

$T_{\alpha}$ being linearly independent homogeneous polynomials and $S_{\alpha}$ in $v$ not identically vanishing polynomials. Then

$$
\overline{\operatorname{degr}}_{u \mid v} P(\varrho u+W(v, q), R(v, q), q) \leqq \max _{\alpha} \overline{\operatorname{degr}}_{u} T_{\alpha}(u) \leqq \overline{\operatorname{degr}}_{u \mid v} P(u, v, q)
$$

\section{Proof of the Auxiliary Power Counting Theorem}

Consider now the integral

$$
\mathscr{I}_{\lambda}(q, \mu)=\int^{\lambda, \mathscr{L}} d^{4} k_{1} \ldots d^{4} k_{m} \frac{\min _{i \in I} \lambda^{-p_{\imath}}\left|P_{i}(k, q)\right|}{E(k, q, \mu)},
$$

where the external momenta $q$ are fixed or at least bounded, and $E(k, q, \mu)$ is given in (5.3). We prove the auxiliary power counting theorem by induction on $m$. For $m=0$ nothing has to be shown. Given some natural number $m_{0}$, we assume the theorem is valid for $m<m_{0}$ and proceed to show that it then also holds for $m=m_{0}$. Let $r=\sum_{i \in I} r_{i}$, where $r_{i}$ is the degree of $P_{i}$ in $k$. 
The proof idea is as follows (cf. [1]). The integral (9.1) will be divided into a sum of integrals over appropriate subsections. These integrals will be splitted into a four-dimensional "outer" and a 4( $\left.m_{0}-1\right)$-dimensional "inner" integration, and the denominator will be made homogeneous by use of Lemma 8.9. The idea is to apply the hypothesis of induction to the inner integrals in such a way that the remaining integrations show the desired cutoff dependence. To this end, the numerator is decomposed into homogeneous components. This allows an appropriate scaling of inner momenta by the outer one (here homogeneity of the denominator is important). After scaling, the inner integrals can be brought to a form similar to the original one. These results are summarized in Lemma 9.1. Lemma 9.2 states that the hypothesis of induction can be applied to these integrals, and combining both lemmas, a simple calculation leads to the desired cutoff dependence of (9.1), i.e., the auxiliary power counting theorem holds for $m=m_{0}$.

In the following we identify $m$ with a given natural number $m_{0}$. We again use the shorthand notation $K_{i}(k)=l_{i}(k, 0)$. At first, for every $\xi=1, \ldots, N$ we define a nonsingular linear transformation

$$
t_{i}=\sum_{j=1}^{m}\left(A_{\xi}\right)_{i j} k_{j} ; \quad\left(A_{\xi}\right)_{i j} \in \mathbf{R} ; \quad i, j=1, \ldots, m
$$

such that

$$
t_{1}=K_{\xi}(k)
$$

Then $k(t)=A_{\xi}^{-1} t$. Without loss of generality we assume $\operatorname{det}\left(A_{\xi}\right)=1$. Furthermore, we introduce the following notations.

i) Let $\mathscr{H}^{0}$ be the set of all $H \in \mathscr{H}$ which are parametrized by a basis of $\mathscr{L}^{5}$. Set $\Delta=\max _{H \in \mathscr{H}^{0}} \delta(H)$. Then we define $\omega_{0}$ by [cf. (5.3)]

$$
\omega_{0}=4 m+\Delta-2 \sum_{\mathscr{N}} n_{j}=\max _{H \in \mathscr{H}^{\circ}} \omega(H) .
$$

ii) Let $\xi \in\{1, \ldots, N\}$. For every sequence $H_{1}, \ldots, H_{s}$ of Zimmermann subspaces which is ordered with respect to a basis of $\mathscr{L}$ containing $l_{\xi}$ and satisfying

a)

b) $\quad H_{s}$ has all line momenta of a basis variable ,

there exists an $i \in I$, so that (5.8) holds. The set of all these $i \in I$ is denoted by $I(\xi)$.

iii) For given $\xi$ define $\mathscr{L}_{\xi}=\left\{\left.l_{j}\left(A_{\xi}^{-1} t, 0\right)\right|_{t_{1}=0} \mid j \in\{1, \ldots, N\} \backslash\{\xi\}\right\}$.

Lemma 9.1. There exist $K>0$ and $R>0$ such that the following statement holds. For all $b>R$ one can find $c(\mu, r, b)>0$, so that

$$
\mathscr{I}_{\lambda}(q, \mu) \leqq c(\mu, r, b) \sum_{\xi=1}^{N} \sum_{y \in Y(\xi)} \int_{W(\lambda)} d^{4} t_{1}\left\|t_{1}\right\| \|^{\omega_{0}-4} \cdot I_{\lambda^{\prime} \xi y}\left(t_{1}, q\right)
$$

\footnotetext{
${ }^{5}$ This means that all momenta of a basis are variable on $H$
} 
for all $\lambda>K$, where $W(\lambda)=\left\{t_{1} \in \mathbf{R}^{4} \mid 1 \leqq\left\|t_{1}\right\| \leqq \lambda+K\right\}, Y(1), \ldots, Y(N)$ are finite sets, and

$$
I_{\lambda^{\prime} \zeta y}\left(t_{1}, q\right)=\int^{\lambda^{\prime}, \mathscr{L}_{\xi}} d^{4} t_{2} \ldots d^{4} t_{m} \frac{\min _{i \in I(\xi)}\left(\lambda^{\prime}\right)^{-p_{2}}\left|P_{i y}\left(A_{\xi}^{-1} t, q\right)\right|}{\left.E\left(A_{\xi}^{-1} t, 0, \mu\right)\right|_{t_{1}=0}} .
$$

Here $\lambda^{\prime}=b(\lambda+d) /\left\|t_{1}\right\|+e$, and

where

$$
P_{i y}\left(A_{\xi}^{-1} t, q\right)=P_{i}\left(A_{\xi}^{-1}\left[F_{i y}(t)\right], q\right),
$$

$$
\left[F_{i y}(t)\right]=\left(c_{i y}, \varrho_{i y} t_{2}, \ldots, \varrho_{i y} t_{m}\right)
$$

$d, e$ and $c_{i y}, \varrho_{i y}>0$ are appropriate constants.

As will be seen below, the hypothesis of induction can be applied to (9.5b) if $b$ is large enough. Remember that $q$ is fixed or at least bounded.

Proof. 1. Applying Lemma 8.9.3 and Lemma 8.9.2 to (9.1), one can find $K>0$ and $c_{1}(\mu, r)>0$ such that for $\lambda>K$,

$$
\mathscr{I}_{\lambda}(q, \mu) \leqq c_{1}(\mu, r) \int_{D^{0,1}}^{\bar{\lambda}, \overline{\mathscr{L}}} d^{4} k_{1} \ldots d^{4} k_{m} \frac{\min _{i \in I}(\bar{\lambda})^{-p_{i}}\left|P_{i}(k, q)\right|}{E(k, 0,0)},
$$

where $\bar{\lambda}=\lambda+K, \overline{\mathscr{L}}=\left\{K_{i}(k) \mid i=1, \ldots, N\right\}$ and $D^{0,1}$ is defined in (8.2). The denominator in (9.7) is homogeneous, and the poles are excluded from the integration range. For every $\xi=1, \ldots, N$ we define a sector $X_{\xi} \subseteq \mathbf{R}^{4 m}$ in the integration domain by

$$
K_{i}^{2}(k) \geqq K_{\xi}^{2}(k) \geqq 1 \quad \text { for all } i=1, \ldots, N
$$

Using $D^{0,1}=\bigcup_{\xi=1}^{N} X_{\xi}$, we get

$$
\mathscr{I}_{\lambda}(q, \mu) \leqq c_{1}(\mu, r) \sum_{\xi=1}^{N} \mathscr{K}_{\bar{\lambda}}\left(q, X_{\xi}\right)
$$

where

$$
\mathscr{K}_{\bar{\lambda}}\left(q, X_{\xi}\right)=\int_{X_{\xi}}^{\bar{\lambda}, \overline{\mathscr{L}}} d^{4} k_{1} \ldots d^{4} k_{m} \frac{\min _{i \in I}(\bar{\lambda})^{-p_{\imath}}\left|P_{i}(k, q)\right|}{E(k, 0,0)} .
$$

In the following let $\xi \in\{1, \ldots, N\}$ be arbitrary. We apply the transformation (9.2) to $\mathscr{K}_{\bar{\lambda}}\left(q, X_{\xi}\right)$. Then

$$
\mathscr{K}_{\bar{\lambda}}\left(q, X_{\xi}\right)=\int_{U(\bar{\lambda})} d^{4} t_{1} \cdot \int_{V(\bar{\lambda}, \xi)} d^{4} t_{2} \ldots d^{4} t_{m} \frac{\min _{i \in I}(\bar{\lambda})^{-p_{i}}\left|P_{i}\left(A_{\xi}^{-1} t, q\right)\right|}{E\left(A^{-1} t, 0,0\right)},
$$

where

$$
U(\bar{\lambda})=\left\{t_{1} \in \mathbf{R}^{4} \mid 1 \leqq\left\|t_{1}\right\| \leqq \bar{\lambda}\right\},
$$

$V(\bar{\lambda}, \xi)=\left\{\left(t_{2}, \ldots, t_{m}\right) \in \mathbf{R}^{4(m-1)} \mid \bar{\lambda}^{2} \geqq K_{i}^{2}\left(A_{\xi}^{-1} t\right) \geqq t_{1}^{2} \quad\right.$ for all $\left.\quad i \in\{1, \ldots, N\} \backslash\{\xi\}\right\}$. 
2. For every $i \in I$ the polynomial $P_{i}$ is decomposed according to

$$
P_{i}\left(A_{\xi}^{-1} t, q\right)=\sum_{\alpha=0}^{r_{l}} T_{i \alpha}\left(A_{\xi}^{-1} t, q\right), \quad i \in I,
$$

where $T_{i \alpha}$ are homogeneous polynomials in the components of $t_{2}, \ldots, t_{m}$ of degree $\alpha$. Further,

$$
T_{i \alpha}\left(A_{\xi}^{-1} t, q\right)=\sum_{\beta_{1}, \ldots, \beta_{4}} T_{i \alpha \beta}\left(A_{\xi}^{-1} t, q\right)
$$

where $\sum_{j=1}^{4} \beta_{j} \leqq r_{i}-\alpha$ and $T_{i \alpha \beta}$ are homogeneous in the components of $t_{1}$, i.e.,

$$
T_{i \alpha \beta}\left(A_{\xi}^{-1} t, q\right)=\left(t_{1}\right)_{1}^{\beta_{1}} \ldots\left(t_{1}\right)_{4}^{\beta_{4}} S_{i \alpha \beta}\left(t_{2}, \ldots, t_{m}, q\right) .
$$

Using the notation of Appendix B and writing $(\alpha \beta)=\left(\alpha_{i}, \beta_{i}\right)_{i \in I}$, we get

$$
\begin{gathered}
\mathscr{K}_{\bar{\lambda}}\left(q, X_{\xi}\right) \leqq \\
\sum_{\alpha_{i},\left(\beta_{1}\right)_{1}, \ldots\left(\beta_{i}\right)_{4} \text { for all } i \in I} \mathscr{J}_{\overline{\hat{\lambda}}(\alpha \beta)}\left(q, X_{\xi}\right), \\
\mathscr{J}_{\bar{\lambda}(\alpha \beta)}\left(q, X_{\xi}\right)=\int_{U(\bar{\lambda})} d^{4} t_{1} \cdot \int_{V(\bar{\lambda}, \xi)} d^{4} t_{2} \ldots d^{4} t_{m} \frac{\min _{i \in I}(\bar{\lambda})^{-p_{t}}\left|T_{i \alpha_{l} \beta_{i}}\left(A_{\xi}^{-1} t, q\right)\right|}{E\left(A_{\xi}^{-1} t, 0,0\right)} .
\end{gathered}
$$

Substituting

$$
\left(t_{2}, \ldots, t_{m}\right)=\left(t_{2}^{\prime}, \ldots, t_{m}^{\prime}\right) \cdot\left\|t_{1}\right\|
$$

in the inner integral and writing $t_{1}^{\prime}=t_{1} /\left\|t_{1}\right\|$ and $\tilde{\lambda}=b \bar{\lambda} /\left\|t_{1}\right\|$, where $b \geqq 1$ (to be chosen below), we get

$$
\mathscr{J}_{\bar{\lambda}(\alpha \beta)}\left(q, X_{\xi}\right) \leqq c^{\prime}(b) \int_{U(\lambda)} d^{4} t_{1}\left\|t_{1}\right\|^{\omega_{0}-4} \cdot \mathscr{J}_{\bar{\lambda}(\alpha \beta)}^{\text {int }}\left(t_{1}, q, X_{\xi}\right),
$$

where $c^{\prime}(b)$ is some power of $b, \omega_{0}$ is defined in (9.3), and

$$
\begin{gathered}
\mathscr{J} \frac{\mathrm{int}}{\bar{\lambda}(\alpha \beta)}\left(t_{1}, q, X_{\xi}\right)=\int_{\nabla(\tilde{\lambda}, \xi)} d^{4} t_{2}^{\prime} \ldots d^{4} t_{m}^{\prime} \frac{\min _{i \in I(\xi)}(\tilde{\lambda})^{-p_{i}}\left|T_{i \alpha_{i} \beta_{t}}\left(A_{\xi}^{-1} t^{\prime}, q\right)\right|}{E\left(A_{\xi}^{-1} t^{\prime}, 0,0\right)}, \quad \text { (9.17b) } \\
\tilde{V}(\tilde{\lambda}, \xi)=\left\{\left(t_{2}^{\prime}, \ldots, t_{m}^{\prime}\right) \in \mathbf{R}^{4(m-1)} \mid \tilde{\lambda}^{2} \geqq K_{i}^{2}\left(A_{\xi}^{-1} t^{\prime}\right) \geqq 1 ; \quad \text { for all } \quad i \in\{1, \ldots, N\} \backslash\{\xi\}\right\} .
\end{gathered}
$$

Here we have used that, by definition of $I(\xi)$ and $\Delta$, for every $i \in I(\xi)$ the inequalities $\alpha_{i}+\sum_{j=1}^{4}\left(\beta_{i}\right)_{j} \leqq r_{i}$ and $r_{i}-p_{i} \leqq \Delta$ hold, i.e., for $\left\|t_{1}\right\| \geqq 1$ and all $i \in I(\xi)$,

$$
(\bar{\lambda})^{-p_{\imath}}\left\|t_{1}\right\|\left(\alpha_{i}+\sum_{j=1}^{4}\left(\beta_{1}\right)_{j}\right)-\Delta \leqq(\tilde{\lambda})^{-p_{t}} \cdot c^{\prime}(b) .
$$

From Lemma 8.5 and (9.13), we get

$$
\mathscr{J} \dot{J}_{\lambda(\alpha \beta)}^{\mathrm{int}}\left(t_{1}, q, X_{\xi}\right) \leqq c_{2}(\mu) \int^{\tilde{\lambda}, \tilde{\mathscr{L}}_{\zeta}} d^{4} t_{2}^{\prime} \ldots d^{4} t_{m}^{\prime} \frac{\min _{i \in I(\xi)}(\tilde{\lambda})^{-p_{\imath}}\left|S_{i \alpha_{i} \beta_{\imath}}\left(t_{2}^{\prime}, \ldots, t_{m}^{\prime}, q\right)\right|}{E\left(A_{\xi}^{-1} t^{\prime}, 0, \mu\right)},
$$


where $\tilde{\mathscr{L}}_{\xi}=\left\{K_{j}\left(A_{\xi}^{-1} t^{\prime}\right) \mid j \in\{1, \ldots, N\} \backslash\{\xi\}\right\} . t_{1}^{\prime}$ is bounded. Using Lemma 8.9.3, there exist $R>0$ and $c_{3}(\mu)>0$, so that for $b>R(\Rightarrow \tilde{\lambda}>R)$

$$
\mathscr{J} \underset{\lambda(\alpha \beta)}{\mathrm{int}}\left(t_{1}, q, X_{\xi}\right) \leqq c_{3}(\mu) \int^{\lambda^{\prime}, \mathscr{L}_{\xi}^{\prime}} d^{4} t_{2}^{\prime} \ldots d^{4} t_{m}^{\prime} \frac{\min _{i \in I(\xi)}\left(\lambda^{\prime}\right)^{-p_{\imath}}\left|S_{i \alpha_{i} \beta_{i}}\left(t_{2}^{\prime}, \ldots, t_{m}^{\prime}, q\right)\right|}{\left.E\left(A_{\xi}^{-1} t^{\prime}, 0, \mu\right)\right|_{t_{1}^{\prime}=0}},
$$

where $\lambda^{\prime}=b \bar{\lambda} /\left\|t_{1}\right\|+R$ and $\mathscr{L}_{\xi}^{\prime}=\left\{\left.K_{j}\left(A_{\xi}^{-1} t^{\prime}\right)\right|_{t_{1}^{\prime}=0} \mid j \in\{1, \ldots, N\} \backslash\{\xi\}\right\}$.

3. So far we have re-introduced masses in the denominators, and the whole $t_{1}$ dependence of the inner integral is contained in $\lambda^{\prime}$. The last step in proving Lemma 9.1 is to re-introduce the polynomials $P_{i}$. Let $\bar{r}=\max _{i \in I} r_{i}$ and choose $\bar{r}+1$ different points $y_{0}, \ldots, y_{\bar{r}} \in \mathbf{R}$ and set

$$
X=\left\{\left(z_{1}, \ldots, z_{4}\right) \mid z_{i} \in\left\{y_{0}, \ldots, y_{\vec{r}}\right\} \text { for all } i=1, \ldots, 4\right\} .
$$

According to Lemma 8.1, there exists $c_{4}(\mu)>0$ such that

$$
\begin{aligned}
& \mathscr{J} \frac{\mathfrak{i n t}_{\lambda(\alpha \beta)}}{\mathrm{int}}\left(t_{1}, q, X_{\xi}\right) \leqq c_{4}(\mu) \sum_{y_{i} \in X \text { for all } i \in I} \\
& \cdot \int^{\lambda^{\prime}, \mathscr{L}_{\xi}^{\prime}} d^{4} t_{2}^{\prime} \ldots d^{4} t_{m}^{\prime} \frac{\min _{i \in I(\xi)}\left(\lambda^{\prime}\right)^{-p_{i}}\left|T_{i \alpha_{l}}\left(A_{\xi}^{-1} t^{\prime}, q\right)\right|_{t_{1}^{\prime}=y_{i}}}{\left.E\left(A_{\xi}^{-1} t^{\prime}, 0, \mu\right)\right|_{t_{1}^{\prime}=0}} .
\end{aligned}
$$

Similar, let $\gamma_{0}, \ldots, \gamma_{\vec{r}}>0$ be $\bar{r}+1$ different points and $Z=\left\{\gamma_{0}, \ldots, \gamma_{\vec{r}}\right\}$. Using Lemma 8.2 , one can find $c_{5}(\mu)>0$ such that

$$
\begin{aligned}
& \mathscr{J} \frac{\operatorname{int}_{\lambda(\alpha \beta)}}{\mathrm{int}}\left(t_{1}, q, X_{\xi}\right) \leqq c_{5}(\mu) \sum_{y_{i} \in X, \varrho_{\iota} \in Z \text { for all } i \in I} \\
& \cdot \int^{\lambda^{\prime}, \mathscr{L}_{\zeta}^{\prime}} d^{4} t_{2}^{\prime} \ldots d^{4} t_{m}^{\prime} \frac{\min _{i \in I(\xi)}\left(\lambda^{\prime}\right)^{-p_{\imath}}\left|P_{i}\left(A_{\xi}^{-1}\left[T_{y_{\imath} \varrho_{l}}^{\prime}\right], q\right)\right|}{\left.E\left(A_{\xi}^{-1} t^{\prime}, 0, \mu\right)\right|_{t_{1}^{\prime}=0}},
\end{aligned}
$$

where

$$
\left[T_{y_{i} \varrho_{\imath}}^{\prime}\right]=\left(y_{i}, \varrho_{i} t_{2}^{\prime}, \ldots, \varrho_{i} t_{m}^{\prime}\right) .
$$

Collecting indices and using the notation (9.6), we get Lemma 9.1.

We now show that the hypothesis of induction can be applied to the integrals (9.5b).

Lemma 9.2. There exist $K_{0}(\mu, q)>0$ and $c_{0}(\mu, q)>0$, so that for $b>K_{0}(\mu, q)$ for all $\xi$ and all $y \in Y(\xi)$ we have

$$
I_{\lambda^{\prime} \xi y}\left(t_{1}, q\right) \leqq c_{0}(\mu, q) \cdot \begin{cases}1 & \text { if } \bar{\omega}(\xi)<0 \\ \left(\lambda^{\prime}\right)^{-1} \log ^{m-1} \lambda^{\prime} & \text { if } \bar{\omega}(\xi)<0 \text { and } p_{i} \geqq 1 \text { for all } i \in I(\xi) \\ \left(\lambda^{\prime}\right)^{\bar{\omega}(\xi)} \log ^{m-1} \lambda^{\prime} & \text { if } \bar{\omega}(\xi) \geqq 0,\end{cases}
$$

where $\bar{\omega}(\xi)=\max _{H \in \mathscr{H}_{\xi}} \omega(H) . \lambda^{\prime}$ is defined in Lemma 9.1. $\mathscr{H}_{\xi} \subset \mathscr{H}$ is the set of 
Zimmermann subspaces, defined by bases of $\mathscr{L}$ containing $l_{\xi}$ with respect to $k_{1}, \ldots, k_{m}$ and having $l_{\xi}=$ const. If $q$ is bounded and $\left\{\delta(H) \mid H \in \mathscr{H}_{\xi}\right\}$ is independent of $q, K_{0}$ and $c_{0}$ can be chosen to be independent of $q$.

Proof. Let $\xi \in\{1, \ldots, N\}$ be arbitrary and

$$
w_{2}, \ldots, w_{m}
$$

ba an arbitrary basis of $\mathscr{L}_{\xi}$ with respect to $\left(t_{2}, \ldots, t_{m}\right)$ of the form

$$
\begin{gathered}
\check{u}_{1}=l_{i_{1}}^{\prime}, \ldots, \check{u}_{d}=l_{i_{d}}^{\prime}, \\
\check{v_{1}}=l_{j_{1}}^{\prime}, \ldots, \check{v}_{m-1-d}=l_{j_{m-1-d}}^{\prime},
\end{gathered}
$$

where $l_{j}^{\prime}=\left.l_{j}\left(A_{\xi}^{-1} t, 0\right)\right|_{t_{1}=0}$. The variables $(\check{u})$ and constants $(\check{v})$ define a Zimmermann subspace $\check{H}$ of $\left(t_{2}, \ldots, t_{m}\right)$. The set of these $\check{H}$, for arbitrary basis $(9.25)$, is denoted by $\check{\mathscr{H}}_{\xi}$. With every $\check{H} \in \check{\mathscr{H}}_{\xi}$ we associate $H \in \mathscr{H}$ as follows. Let a basis (9.25b) be given. Take the basis

$$
z_{2}, \ldots, z_{m}, l_{\xi}
$$

of $\mathscr{L}$ with respect to $\left(k_{1}, \ldots, k_{m}\right)$ of the form

$$
\begin{aligned}
u_{w} & =l_{i_{w}}(k, q), \quad w=1, \ldots, d, \\
v_{w} & =l_{j_{w}}(k, q), \quad w=1, \ldots, m-1-d, \\
v_{m-d} & =l_{\xi}(k, q),
\end{aligned}
$$

and let $k=A_{\xi}^{-1} T$, where $T=\left(x, t_{2}, \ldots, t_{m}\right)$. Then

$$
\begin{aligned}
u_{w} & =\check{u}_{w}+U_{w}(x, q), \quad w=1, \ldots, d, \\
v_{w} & =\check{v}_{w}+V_{w}(x, q), \quad w=1, \ldots, m-1-d, \\
v_{m-d} & =x+Q_{\xi}(q),
\end{aligned}
$$

where $U_{w}, V_{w}, Q_{\xi}$ are linear functions. Then the $H \in \mathscr{H}$ associated with $\check{H} \in \check{\mathscr{H}}_{\xi}$ is defined by variables $(u)=\left(u_{1}, \ldots, u_{d}\right)$ and constants $(v)=\left(v_{1}, \ldots, v_{m-d}\right)$. We define $\delta(\check{H})=\delta(H)$. The set of these $H \in \mathscr{H}$ is identical to $\mathscr{H}_{\xi}$ in the lemma.

By this construction, to every sequence $\breve{H}_{1}, \ldots, \breve{H}_{s}$ of Zimmermann subspaces in $\check{\mathscr{H}}_{\xi}$, which is ordered with respect to a basis $(9.25)$ corresponds an ordered sequence $H_{1}, \ldots, H_{s}$ of Zimmermann subspaces in $\mathscr{H}_{\xi}$, with respect to the corresponding basis (9.26). Adding $H_{s+1} \in \mathscr{H}$, parametrized by the whole basis (9.26), we again get an ordered sequence. Let $\left(\check{u}^{j}\right)$ be the parameters of $\check{H}_{j}$ and $\left(\check{v}^{j}\right)$ the complement variables, i.e., $\left(\check{u}^{j}, \check{v}^{j}\right)=\left(w_{2}, \ldots, w_{m}\right)$, and correspondingly $\left(u^{j}\right)$ the parameters of $H_{j}$, $\left(u^{j}, v^{j}\right)=\left(z_{2}, \ldots, z_{m}, l_{\xi}\right)$, for every $j=1, \ldots, s$. By construction of $I(\xi)$, using Lemma 8.10 [and inequality (C.4) of Appendix C], there exists an $i \in I(\xi)$ such that

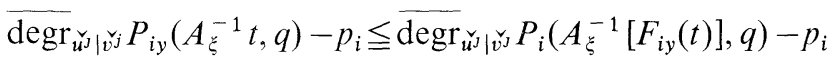

$$
\begin{aligned}
& \leqq \overline{\operatorname{degr}}_{u^{j} \mid v^{j}} P_{i}\left(k\left(u^{j}, v^{j}, q\right), q\right)-p_{i} \\
& \leqq \delta\left(H_{j}\right) \text {, }
\end{aligned}
$$

for all $j=1, \ldots, s$. This means the set $\left\{\delta(\check{H}) \mid \check{H} \in \check{\mathscr{H}}_{\xi}\right\}$ is a UV-set of the numerator 
of (9.5b). For every $\check{H} \in \check{\mathscr{H}}_{\xi}$, parametrized by (9.25b), and corresponding $H \in \mathscr{H}_{\xi}$,

$$
\begin{aligned}
\omega(\breve{H}) & =4 d+\delta(\breve{H})-\left.\overline{\operatorname{degr}}_{\breve{u}} E\left(A_{\xi}^{-1} t, 0, \mu\right)\right|_{t_{1}=0} \\
& =4 d+\delta(H)-\overline{\operatorname{degr}}_{u} E(k(u, v, q), q)=\omega(H) .
\end{aligned}
$$

Thus, all the conditions are met for the auxiliary power counting to apply to the inner integrals $I_{\lambda^{\prime} \xi y}\left(t_{1}, q\right)$. According to the hypothesis of induction, we get Lemma 9.2.

We now proceed to complete the proof of the main Theorem 2. All that remains to do is to insert the cutoff estimates of Lemma 9.2 into the inequality of Lemma 9.1. Then, re-expressing $\lambda^{\prime}$ by $\lambda$, the remaining integration can be done without problems. Choose a fixed $b$ according to Lemma 9.1 and Lemma 9.2. Then there exist constants $\bar{c}(\mu, q)>0$ and $\bar{K}(\mu, q)>0$ such that for all $\lambda>\bar{K}(\mu, q)$,

$$
\begin{aligned}
& \mathscr{I}_{\lambda}(q, \mu) \leqq \bar{c}(\mu, q) \sum_{\xi=1}^{N}
\end{aligned}
$$

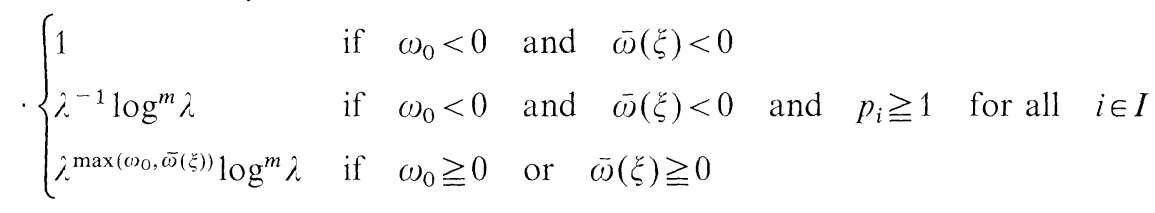

$$
\begin{aligned}
& \leqq \bar{c}(\mu, q) \\
& \begin{cases}1 & \text { if } \max _{H \in \mathscr{H}} \omega(H)<0 \\
\lambda^{-1} \log ^{m} \lambda & \text { if } \max _{H \in \mathscr{H}} \omega(H)<0 \text { and } p_{i} \geqq 1 \text { for all } i \in I \\
\lambda^{\max _{H \in \mathscr{H}}[\omega(H)]} \log ^{m} \lambda & \text { if } \max _{H \in \mathscr{H}} \omega(H) \geqq 0,\end{cases}
\end{aligned}
$$

where we have used

$$
\max \left(\omega_{0}, \bar{\omega}(\xi)\right) \leqq \max _{H \in \mathscr{H}_{\xi}}\left(\max _{H^{\prime} \in \mathscr{H}^{0}} \omega\left(H^{\prime}\right), \omega(H)\right) \leqq \max _{H \in \mathscr{H}} \omega(H) .
$$

$\bar{K}$ and $\bar{c}$ can be chosen to be independent of $q$ if $q$ is bounded and if $\{\delta(H) \mid H \in \mathscr{H}\}$, and hence $\{\omega(H) \mid H \in \mathscr{H}\}$ are independent of $q$. This completely proves the auxiliary power counting theorem.

\section{Conclusion}

We have proved a convergence theorem for Feynman integrals with a lattice cutoff. Under very general conditions, it states existence of the continuum limit as well as its coincidence with the formal limit, i.e., the Feynman integral, which results from taking the continuum limit in the integrand. If convergence holds, only a neighborhood of zero momentum in the Brillouin zone contributes to the limit. 
An important convergence condition is the naturalness of line momenta. This means that their homogeneous parts in the integration momenta $k$,

$$
\sum_{i=1}^{m} C_{i j} k_{j}
$$

satisfy $C_{i j} \in \mathbf{Z}$ for the given representation (3.1) and for every choice of independent line momenta as integration variables $k_{1}, \ldots, k_{m}$. For a lattice Feynman integral it is always possible to choose the loop momenta in such a way that this condition is satisfied, e.g. if the loop momenta $k_{1}, \ldots, k_{m}$ coincide with momenta of lines. However, in the case of renormalizations one must be very careful in order to ensure that the subtracted integrand still satisfies this condition. Note that in the power counting theorem of Hahn and Zimmermann [1] the condition of naturalness is unnecessary. However, this theorem can only be applied to integrals having a rational integrand. On the lattice, in connection with the periodicity of the integrand, naturalness makes sure that only one Brillouin zone contributes in the continuum limit.

Furthermore, the theorem assumes that the propagators have only one pole in the Brillouin zone, located at vanishing line momenta. This means that the denominators of the propagators

$$
\frac{1}{\frac{\eta(l a)}{a^{2}}+\mu^{2}}
$$

satisfy $\eta(l a \neq 0)>0$ in the Brillouin zone. If this condition would be violated, the assumed periodicity of the integrand would not be sufficient for convergence. In particular, the theorem does not apply to lattice fermions with propagators having poles on the boundary of the Brillouin zone. In general, the pole condition implies that only a small neighborhood of zero momentum contributes as the lattice spacing tends to zero, and that the continuum limit of a lattice Feynman integral is equal to the formal limit.

For simplicity, we have always assumed the numerator and denominator of the integrand to be $C^{\infty}$. Actually, the denominator needs to be differentiable only in a small neighbourhood of vanishing line momenta, and globally continuous. In the case of renormalization, the whole integrand has to be differentiable to a degree depending on the divergence degrees.

The main point of the convergence theorem is that it is a power counting theorem. This means that convergence of Feynman integrals in the continuum limit is described by ultraviolet divergence degrees with respect to special subspaces of the integration momenta, called Zimmermann subspaces. In order to get convergence in the continuum limit, the divergence degrees with respect to all these subspaces should be smaller than zero. Due to the structure of diagrams with a lattice cutoff, we have a new kind of degrees to be dinstinguished from UV-degrees of rational functions [1]. A lattice degree describes the behavior of a Feynman integrand for large internal momenta of a Zimmermann subspace and small lattice spacing $a$ simultaneously. To discuss naively large momenta for fixed $a$ would be meaningless because of the periodicity of the integrand. 
To every Zimmermann subspace there corresponds a (sub-)diagram. Hence, loosely speaking, negative UV-divergence degrees mean that all subdiagrams are convergent. Usually, a Feynman diagram must be renormalized. In terms of a power counting theorem this means that counterterms have to be arranged in such a way that divergence degrees of all subspaces are negative. In a following paper [4], this correspondence will be used to construct a renormalization scheme for Feynman integrals on the lattice, which is analogous to the BPHZ finite part prescription for continuum Feynman integrals. It will be seen that counterterms instead of being polynomials are periodic functions. From the fact that negative lattice divergence degrees insure not only the existence of the continuum limit but also its coincidence with the formal limit, it will follow that renormalized perturbation theory is universal, which means that the continuum limit does not depend on a specific choice of the lattice action.

The power counting theorem applies to a wide class of lattice field theories. In this investigation we have been concerned solely with the problem of ultraviolet divergencies. We have assumed all fields to be massive in order to avoid infrared singularities. In the given form the power counting theorem does not apply to lattice field theories with massless propagators. Whereas the lattice provides a UV-cutoff, IR-singularities are expected to be the same as in the continuum. This suggests that one should supplement the UV-power counting conditions by IR-power counting conditions, which describe the behavior of a Feynman integrand for small internal momenta and state IR-convergence at non-exceptional external momenta. By this modification, the power counting theorem should apply also to massless field theories on the lattice. In a forthcoming paper we will show that this is indeed the case, and that the ideas presented here will go through.

\section{Appendix A. Proof of Lemma A.1}

We first show that the propagators of (8.19) are of no importance for the validity of Lemma A.1. Recall that the line momenta $l_{i} \in \mathscr{N}$ are of the form

$$
l_{i}(k, q)=\sum_{j=1}^{m} C_{i j} k_{j}+Q_{i}(q) .
$$

Given compact cubes $\sigma_{1}, \sigma_{2}$ and $\sigma_{2}$ containing an open set, let $\sigma=\sigma_{1} \cup \sigma_{2}$ and choose $K(C, \sigma)>0$ such that for all $l_{i} \in \mathscr{N}$,

and define

$$
\left\|\sum_{j=1}^{m} C_{i j} k_{j}\right\| \leqq K(C, \sigma) \text { for all } k \in \sigma,
$$

$$
g_{\sigma}(Q)=\prod_{\mathscr{N}} \frac{1}{\left(K(C, \sigma)+\left\|Q_{i}\right\|\right)^{2}+\mu_{i}^{2}} .
$$

Using the triangle inequality and Lemma 8.3 one can find a constant $d(C, \sigma, \mu)>0$, so that for all $k \in \sigma$,

$$
g_{\sigma}(Q) \leqq \frac{1}{\prod_{\mathscr{N}}\left(l_{i}^{2}(k, q)+\mu_{i}^{2}\right)} \leqq d(C, \sigma, \mu) g_{\sigma}(Q) .
$$


Now Lemma $A .1$ is a direct consequence of

Lemma A.2. Let $w \in \mathbf{N}, r \in \mathbf{N}_{0}$ and $\sigma_{1}, \sigma_{2}$ compact cubes in $\mathbf{R}^{n}, \sigma_{2}$ containing an open set. Then there is a constant $c\left(\sigma_{1}, \sigma_{2}, r\right)>0$, so that

$$
\int_{\sigma_{1}} d x_{1} \ldots d x_{n} \min _{i=1, \ldots, w}\left|P_{i}(x)\right| \leqq c\left(\sigma_{1}, \sigma_{2}, r\right) \int_{\sigma_{2}} d x_{1} \ldots d x_{n} \min _{i=1, \ldots, w}\left|P_{i}(x)\right|
$$

for arbitrary polynomials $P_{i}$ of degree $r_{i}$ in $x_{1}, \ldots, x_{n}$, with $\sum_{i=1}^{w} r_{i} \leqq r$.

Proof. By complete induction on the number $n$ of integrations.

A. $n=1$. By induction on $r$. The case $r=0$ is trivial. Assume for some $r \in \mathbf{N}_{0}$ there exists a constant $c\left(\sigma_{1}, \sigma_{2}, r\right)>0$, so that

$$
\int_{\sigma_{1}} d x \min _{i=1, \ldots, w}\left|P_{i}(x)\right| \leqq c\left(\sigma_{1}, \sigma_{2}, r\right) \int_{\sigma_{2}} d x \min _{i=1, \ldots, w}\left|P_{i}(x)\right|
$$

for all polynomials $P_{i}$ of a degree $r_{i}, \sum_{i=1}^{w} r_{i} \leqq r$. Now let $P_{i}$ of degree $r_{i}$ and $\sum_{i=1}^{w} r_{i}=r+1$. Suppose every $P_{i}$ has the form

$$
P_{i}(x)=a_{i} \prod_{j=1}^{r_{2}}\left(x-z_{i j}\right),
$$

where $a_{i}, z_{i j} \in \mathbf{C} ; a_{i} \neq 0$. Choose $R(\sigma)>0$, so large, that for all $z \in \mathbf{C},|z|>R(\sigma)$,

$$
\frac{1}{2} \leqq\left|1-\frac{x}{z}\right| \leqq \frac{3}{2} \text { for all } x \in \sigma .
$$

Let $z=\left(z_{11}, \ldots, z_{w r_{w}}\right) \in \mathbf{C}^{r+1}, \quad a=\left(a_{1}, \ldots, a_{w}\right) \in \mathbf{C}^{w}$ and for $\left(r_{1}, \ldots, r_{w}\right) \in \mathbf{N}_{0}^{w}$, $\sum_{i=1}^{w} r_{i}=r+1$

$$
f_{r_{1} \ldots r_{w}}(z, a)=\frac{\int_{\sigma_{1}} d x \min _{i=1, \ldots, w}\left|P_{i}(x)\right|}{\int_{\sigma_{2}} d x \min _{i=1, \ldots, w}\left|P_{i}(x)\right|} .
$$

All $f_{r_{1} \ldots r_{w}}$ are continuous and non-negative. If $\left|z_{i j}\right| \leqq R(\sigma)$ for all $i, j$, then there exists a constant $B\left(\sigma_{1}, \sigma_{2}\right)>0$, so that

$$
f_{r_{1} \ldots r_{w}}(z, a) \leqq B\left(\sigma_{1}, \sigma_{2}\right) .
$$

On the other hand, if $\left|z_{i_{0} j_{0}}\right|>R(\sigma)$ for some $j_{0}, i_{0}$, set

such that

$$
P_{i_{0}}^{\prime}(x)=a_{i_{0}} z_{i_{0} j_{0}} \prod_{j\left(\neq j_{0}\right)}\left(x-z_{i_{0} j}\right)
$$

by (A.6), and set

$$
\frac{1}{2}\left|P_{i_{0}}^{\prime}(x)\right| \leqq\left|P_{i_{0}}(x)\right| \leqq \frac{3}{2}\left|P_{i_{0}}^{\prime}(x)\right| \text { for all } \quad x \in \sigma
$$

Then, by induction hypothesis

$$
P_{i}^{\prime}=P_{i} \text { for all } i \neq i_{0} .
$$

$$
f_{r_{1} \ldots r_{w}}(z, a) \leqq 3 c\left(\sigma_{1}, \sigma_{2}, r\right) .
$$


Choosing $c\left(\sigma_{1}, \sigma_{2}, r+1\right)=\max \left(B\left(\sigma_{1}, \sigma_{2}\right), 3 c\left(\sigma_{1}, \sigma_{2}, r\right)\right)$ the assertion follows for $n=1$.

B. Let $n>1$. Assume the lemma holds for all natural numbers $v<n$. Without loss of generality set $\sigma_{1}=[a, b]^{n}$ and $\sigma_{2}=[\bar{a}, \bar{b}]^{n}$. By induction hypothesis, there exist $c_{1}(a b \bar{a} \bar{b})>0$ and $c_{n-1}(a b \bar{a} \bar{b})>0$, so that for all polynomials $P_{i}$ of degree smaller or equal to $r_{i}$ in $x_{1}, \ldots, x_{n}$, where $\sum_{i=1}^{w} r_{i} \leqq r$, using Fubini's theorem

$$
\begin{aligned}
& \int_{a}^{b} d x_{1} \int_{a}^{b} d x_{2} \ldots \int_{a}^{b} d x_{n} \min _{i=1, \ldots, w}\left|P_{i}\left(x_{1}, \ldots, x_{n}\right)\right| \\
& \leqq c_{n-1} \int_{a}^{b} d x_{1} \int_{\bar{a}}^{\bar{b}} d x_{2} \ldots \int_{\bar{a}}^{\bar{b}} d x_{n} \min _{i=1, \ldots, w}\left|P_{i}\left(x_{1}, \ldots, x_{n}\right)\right| \\
& \leqq c_{1} c_{n-1} \int_{\bar{a}}^{\bar{b}} d x_{1} \ldots \int_{\bar{a}}^{\bar{b}} d x_{n} \min _{i=1, \ldots, w}\left|P_{i}\left(x_{1}, \ldots, x_{n}\right)\right| .
\end{aligned}
$$

This proves Lemma A.2.

\section{Appendix B. A Useful Inequality}

We state a simple but useful inequality.

Lemma B.1. Let $n \in \mathbf{N}$ be a natural number, $I=\{1, \ldots, n\}$, and $L_{i}$ a finite set for every $i \in I$. For all $i \in I$ and all $l \in L_{i}$, let $x_{l} \geqq 0$. Then

$$
\min _{i \in I} \sum_{l \in L_{l}} x_{l} \leqq \sum_{l_{1} \in L_{1}} \ldots \sum_{l_{n} \in L_{n}} \min _{i \in I} x_{l_{l}}
$$

This inequality can be written in a more concise form. For $I=\left\{i_{1}, \ldots, i_{n}\right\}$ and sets $L_{i_{1}}, \ldots, L_{i_{n}}$ define

$$
L=\bigotimes_{i \in I} L_{i}=\left(L_{i_{1}}, \ldots, L_{i_{n}}\right)
$$

i.e., every $l \in L$ is of the form

where

$$
l=\left(l_{i}\right)_{i \in I}=\left(l_{i_{1}}, \ldots, l_{i_{n}}\right)
$$

$$
l_{i_{k}} \in L_{i_{k}}, \quad k=1, \ldots, n
$$

Using this notation, Lemma B.1 can be written as follows.

Lemma B.2. Let $I$ and $L_{i}$ for every $i \in I$ be finite sets. Let $x_{l} \geqq 0$ for all $l \in L_{i}$ and $i \in I$. Then

$$
\min _{i \in I} \sum_{l \in L_{l}} x_{l} \leqq \sum_{l \in L} \min _{i \in I} x_{i l}
$$

where $L=\otimes_{i \in I} L_{i}$, and for every $l=\left(l_{i}\right)_{i \in I}: x_{i l}=x_{l_{l}}$. 


\section{Appendix C. UV-Degrees of Polynomials}

Let $P$ be a polynomial of $u, v$ and $q$. The UV-degree $\overline{\operatorname{degr}}_{u \mid v} P(u, v, q)$ is defined as follows. $P$ can be written as

$$
P(u, v, q)=\sum_{\alpha} Q_{\alpha}(v, q) M_{\alpha}(u), \quad Q_{\alpha}(v, q) \neq 0 \quad \text { in } \quad v(q \text { fixed }),
$$

where $M_{\alpha}$ are linearly independent homogeneous polynomials in $u$, and $Q_{\alpha}$ are polynomials. Then we define

$$
\overline{\operatorname{degr}}_{u \mid v} P \equiv \max _{\alpha} \operatorname{degr} M_{\alpha},
$$

$\operatorname{degr} M_{\alpha}$ being the homogeneity degree of $M_{\alpha}$. Usually, all parameters which are

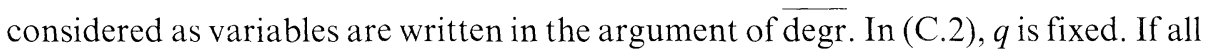
momenta are variables we will sometimes use the shorthand notation

In general,

$$
\overline{\operatorname{degr}}_{u} P(u, v, q) \equiv \overline{\operatorname{degr}}_{u \mid v q} P(u, v, q) .
$$

$$
\overline{\operatorname{degr}}_{u \mid v} P(u, v, q) \leqq \overline{\operatorname{degr}}_{u} P(u, v, q) .
$$

A useful characterization is the following. $\overline{\operatorname{degr}}_{u \mid v} P(u, v, q)=\delta$ if and only if

$$
P(\lambda u, v, q)=A(u, v, q) \cdot \lambda^{\delta}+O\left(\lambda^{\delta-1}\right), \quad \lambda \rightarrow \infty,
$$

$A(u, v, q) \neq 0$ in $u, v(q$ fixed!).

\section{Appendix D. Naturalness of Line Momenta}

We state an important property of a natural set of line momenta. This property is needed when the integration domain of a Feynman integral on the lattice is divided into various sections to determine the continuum limit behavior. It happens that line momenta have values in neighborhoods of poles of propagators in higher BZ's. The following two lemmas show that, if the neighborhoods are chosen sufficiently small, it is possible to shift the line momenta into the first BZ simultaneously by a translation of the integration momenta by reciprocal lattice vectors. Under such a transformation, the periodic numerator of a Feynman integrand does not change.

We shall use the notation of Definition 3.1.

Lemma D.1. Given a set $\mathscr{L}=\left\{l_{1}, \ldots, l_{N}\right\}$ of four-momenta, there exist $\varepsilon>0$ and $a_{0}>0$, so that for all $a<a_{0}$ the following statement holds:

Let $J \subseteq\{1, \ldots, N\}$ and $z=\left\{z_{i} \in \mathbf{Z}^{4} \mid i \in J\right\}$ such that

$$
\begin{aligned}
M_{J z}(\varepsilon, a)= & \left\{\left(k_{1}, \ldots, k_{m}\right) \in[-\pi / a, \pi / a]^{4 m} \mid\left\|l_{i}(k, q)-(2 \pi / a) z_{i}\right\|<(\pi / a) \varepsilon,\right. \\
& \text { for all } i \in J\}
\end{aligned}
$$

is not empty. Then there exists a momentum configuration $k \in[-\pi / a, \pi / a]^{4 m}$ such that

$$
K_{i}(k)=\frac{2 \pi}{a} z_{i} \quad \text { for all } \quad i \in J
$$


If the statement holds for some $\varepsilon>0$, then so for $\varepsilon^{\prime}, 0<\varepsilon^{\prime} \leqq \varepsilon$. The lemma states that neighborhoods of the poles can always be chosen so small that their intersection (D.1) with the integration domain is non-empty only if the "internal" momenta $K_{i}$, for some $k$, satisfy (D.2).

Proof. Let $J \subseteq\{1, \ldots, N\}$ be an arbitrary subset and $Z=\left\{z \in \mathbf{Z}^{4}|\|z\|<1+2 m| C \mid\right\}$, where $|C|=\max \left|C_{i j}\right|$ (cf. Definition 3.1).

1. Set $a_{1}=\min _{i=1, \ldots, n}\left(\pi, Q_{i} \|\right)$ and $\varepsilon_{1}=1 / 2$. Then $M_{J_{z}}(\varepsilon, a)=\emptyset$ if $\varepsilon<\varepsilon_{1}, a<a_{1}$, and $z_{1} \notin Z$ for some $i \in J$. For, a simple calculation shows

$$
\left\|l_{i}(k, q)-\frac{2 \pi}{a} z_{i}\right\| \geqq \frac{\pi}{a}\left(2\left\|z_{i}\right\|-(4 m|C|+1)\right)>\frac{\pi}{a} \varepsilon .
$$

2. Let $M_{J z}(\varepsilon, a) \neq \emptyset$. If there exists no $k \in[-\pi / a, \pi / a]^{4 m}$ satisfying (D.2), then there exist $j \in J$ and $\delta>0$ such that

$$
\left\|K_{j}(k)-\frac{2 \pi}{a} z_{j}\right\| \geqq \frac{\pi}{a} \delta \text { for all } k \in[-\pi / a, \pi / a]^{4 m} .
$$

This means

$$
\left\|l_{j}(k, q)-\frac{2 \pi}{a} z_{j}\right\| \geqq \frac{\pi}{a} \delta-\left\|Q_{j}\right\|>\frac{\pi}{a} \varepsilon(J, z)
$$

if $a<a_{2}(J, z)=\pi \delta /\left(2\left\|Q_{j}\right\|\right)$ and $\varepsilon<\varepsilon_{2}=\delta / 4$, in contradiction to $M_{J_{z}}(\varepsilon, a) \neq \emptyset$. Taking the minimum of all $\varepsilon_{1}, \varepsilon_{2}(J, z)$ and of all $a_{2}(J, z), a_{1}$, respectively, the assertion follows.

The importance of Lemma D.1 rests on the following

Lemma D.2. Let the set $\mathscr{L}=\left\{l_{1}, \ldots, l_{N}\right\}$ be natural and $J \subseteq\{1, \ldots, N\}$ an arbitrary subset. If $k \in \mathbf{R}^{4 m}$ exists, satisfying

$$
K_{i}(k)=\frac{2 \pi}{a} z_{i} \text { for some } z_{i} \in \mathbf{Z}^{4} \text { and all } i \in J,
$$

then there exist reciprocal lattice vectors

$$
\Delta_{1}, \ldots, \Delta_{m} \in\left\{\frac{2 \pi}{a} r \mid r \in \mathbf{Z}^{4}\right\}
$$

so that for $\Delta=\left(\Delta_{1}, \ldots, \Delta_{m}\right)$

$$
K_{i}(\Delta)=\frac{2 \pi}{a} z_{i} \text { for all } i \in J
$$

The translation alluded to in the introduction to this appendix thus consists in

so that for all $i \in J$

$$
k_{j} \rightarrow k_{j}+\Delta_{j}, \quad j=1, \ldots, m,
$$

$$
l_{i} \rightarrow l_{i}+\frac{2 \pi}{a} z_{i} .
$$


Proof. Let $J \subseteq\{1, \ldots, N\}$ and $k \in \mathbf{R}^{4 m}$, satisfying

$$
K_{i}(k)=\frac{2 \pi}{a} z_{i}, \quad z_{i} \in \mathbf{Z}^{4} \text { for all } i \in J
$$

Choose linearly independent $K_{c_{1}}, \ldots, K_{c_{m}}$, so that $K_{c_{1}}, \ldots, K_{c_{d}}, d \leqq m$, is a basis of $\left\{K_{i} \mid i \in J\right\}$. According to Definition 3.1

for every $j=1, \ldots, m$, and

$$
k_{j}=\sum_{l=1}^{m} A_{j l} K_{c_{l}}, \quad A_{j l} \in \mathbf{Z},
$$

$$
K_{i}=\sum_{l=1}^{d} D_{i l} K_{c_{l}} \quad \text { for all } \quad i \in J ; \quad D_{i l} \in \mathbf{Z} .
$$

Define for $j=1, \ldots, m$

$$
\Delta_{j}=\frac{2 \pi}{a} \sum_{l=1}^{d} A_{j l} z_{c_{l}} \in \frac{2 \pi}{a} \mathbf{Z}^{4}
$$

and $\Delta=\left(\Delta_{1}, \ldots, \Delta_{m}\right)$. Then, for every $i \in J$

$$
K_{i}(\Delta)=\sum_{l=1}^{d} D_{i l} K_{c_{l}}(\Delta)=\sum_{l=1}^{d} D_{i l} K_{c_{l}}(k)=K_{i}(k)=\frac{2 \pi}{a} z_{i}
$$

Acknowledgement. I am grateful to M. Lüscher for proposing this work and for many helpful discussions.

\section{References}

1. Hahn, Y., Zimmermann, W.: An elementary proof of Dyson's power counting theorem. Commun. Math. Phys. 10, 330-342 (1968)

2. Weinberg, S.: High energy behaviour in quantum field theory. Phys. Rev. 118, 838-849 (1960)

3. Nakanishi, N.: Graph theory and Feynman integrals. New York: Gordon and Breach 1971

4. Reisz, T.: Renormalization of Feynman integrals on the lattice. Commun. Math. Phys. (in press)

Communicated by A. Jaffe

Received September 10, 1987 